Pre-print (Computational Materials Science) - accepted in April 2018

\title{
Designing nanoindentation simulation studies by appropriate indenter choices: \\ Case study on single crystal tungsten
}

Saurav Goel $^{1 *}$, Graham Cross ${ }^{2,3}$, Alexander Stukowski ${ }^{4}$, Ernst Gamsjäger ${ }^{5}$, Ben Beake ${ }^{6,7}$ and Anupam Agrawal ${ }^{8}$

${ }^{I}$ School of Aerospace, Transport and Manufacturing, Cranfield University, Bedfordshire, MK430AL, UK

${ }^{2}$ Adama Innovations Limited, Dublin 2, Ireland

${ }^{3}$ CRANN Nanoscience Institute, School of Physics, Trinity College, Dublin 2, Ireland

${ }^{4}$ Institute of Materials Science, Darmstadt University of Technology, Darmstadt, D-64287, Germany

${ }^{5}$ Institute of Mechanics, Montanuniversität Leoben, Leoben 8700, Austria

${ }^{6}$ Micro Materials Limited, Willow House, Yale Business Village, Ellice Way, Wrexham, LL13 7YL, UK

${ }^{7}$ School of Mechanical Engineering, University of Leeds, UK

${ }^{8}$ Mays Business School, Texas A\&M University, College Station, TX, USA

*Corresponding author Tel.: +44-1234-754132, Email address: saurav.goel@cranfield.ac.uk

\begin{abstract}
:
Atomic simulations are widely used to study the mechanics of small contacts for many contact loading processes such as nanometric cutting, nanoindentation, polishing, grinding and nanoimpact. A common assumption in most such studies is the idealisation of the impacting material (indenter or tool) as a perfectly rigid body. In this study, we explore this idealisation and show that active chemical interactions between two contacting asperities lead to significant deviations of atomic scale contact mechanics from predictions by classical continuum mechanics. We performed a testbed study by simulating velocity-controlled, fixed displacement nanoindentation on single crystal tungsten using five types of indenter (i) a rigid diamond indenter (DI) with full interactions, (ii) a rigid indenter comprising of the atoms of the same material as that of the substrate i.e. tungsten atoms (TI), (iii) a rigid diamond indenter with pairwise attraction turned off, (iv) a deformable diamond indenter and (v) an imaginary, ideally smooth, spherical, rigid and purely repulsive indenter (RI). Corroborating
\end{abstract}


Pre-print (Computational Materials Science) - accepted in April 2018

the published experimental data, the simulation results provide a useful guideline for selecting the right kind of indenter for atomic scale simulations.

Keywords: MD simulation; indenter; nanoindentation; deformation; tungsten.

\section{Abbreviations:}

$\begin{array}{ll}A B O P & \text { Analytical bond order potential } \\ B C C & \text { Body centred cubic } \\ C A T & \text { Crystal analysis tool (software program) } \\ D X A & \text { Dislocation extraction algorithm } \\ M D & \text { Molecular dynamics } \\ N V E & \text { Microcanonical ensemble } \\ \text { OVITO } & \text { Open Visualization tool (software program) } \\ \text { PBC } & \text { Periodic boundary condition } \\ \text { TEM } & \text { Transmission electron microscope }\end{array}$

\section{Nomenclatures:}

$a$

A

$b$

B

DI

TI

RI

$\mathrm{C}_{\mathrm{ij}}$

DI-NA

$E$

$E_{r}$

$\mathrm{F}$ or $\mathrm{P}$

$\mathrm{F}(\mathrm{r})$

G

$\mathrm{h}$

$\mathrm{h}_{\mathrm{f}}$

$\mathrm{h}_{\max }$

$H$

$\mathrm{K}$
Contact radius of the spherical indenter

Projected area

Direction of Burgers vector

Bulk modulus

Simulation using a rigid diamond indenter

Simulation using a rigid tungsten indenter

Simulation with a repulsive indenter (force potential)

Elastic constants of the material

Diamond indenter with no pairwise attraction with the substrate

Elastic modulus of the material

Reduced elastic modulus of the material system

Normal force or load on the indenter

Repulsive force potential

Shear modulus (GPa)

Instantaneous displacement of the indenter

Residual depth of indentation

Maximum depth of indentation

Hardness of the material

Force constant 
Pre-print (Computational Materials Science) - accepted in April 2018

$\mathrm{L}$

$P-h$

$\mathrm{p}_{\mathrm{m}}$

$R$

$\mathrm{R}_{\mathrm{pl}}$

$r$

$\mathrm{r}_{0}$

$\mathrm{S}$

W

$\sigma_{\text {hydrostatic }}$

$\sigma_{1}, \sigma_{2}$ and $\sigma_{3}$

$\varepsilon$

$\tau$

Total length of dislocations in $\AA$.

Load-displacement curve

Contact pressure

Radius of the indenter

Radius of the plastic zone

Distance between two dimers

Cutoff radius between indenter and the substrate material

Slope of the unloading curve

Tungsten

Hydrostatic stress

Principal stresses

Strain

Shear stress

\section{Introduction}

The majority of nanoindentation studies have focused on investigating the deformation of the substrate and the indenter is often considered as a smooth, rigid and otherwise inert body impenetrable by other bodies. This inert body assumption stems from the theory of continuum mechanics and usually the atomic description of the indenter is not taken into account. While this assumption is widespread in the atomistic simulation studies of nanoindentation $[1,2]$, nanometric cutting or nanoscratching [3, 4], there are some notable exceptions. These include MD simulations of nanoindentation of a gold substrate using a nickel tip [5, 6], of a tungsten substrate using a deformable tungsten indenter [7], of aluminum using infinitely hard nickel [8] and of other engineering materials such as $\mathrm{CaF}_{2}$, NiTi and gold using a hypothetical, purely repulsive type of spherical force field [9$11]$.

Recently, the fundamental mechanism between dislocations and a single nanotwin was elucidated by in situ transmission electron microscopy (TEM) nanoindentation [12]. High speed scratching or grinding using a single diamond tip are proposed [13], to address the fundamental mechanisms for abrasive machining, such as grinding, polishing and lapping. In these processes, as the chemical interaction changes with each type of indenter, the resulting magnitude and direction of the force 
Pre-print (Computational Materials Science) - accepted in April 2018

being transferred by the indenter to the substrate for a given displacement differs. The implication of this change is not quantified in extant research. Our purpose in this study is to examine the specific role of tip-sample interaction perturbations on continuum contact mechanics concepts using state-ofthe-art, fully atomistic molecular dynamics modelling. We focus on how modeling indenters with different material systems can affect the forces at an atomic level. Our exploration is important for understanding how the differences in adhesion (clinging between two different atomic species) and cohesion (clinging between the same atomic species) governs the initial contact and what subsequent effects it has on the contact mechanics at the meso and macro scales.

\section{Literature review}

The word Tungsten is derived from the Swedish words 'tung sten' meaning 'heavy stone' while its chemical symbol $W$ comes from the German name Wolfram. Scheelite and Wolframite are the two naturally occurring tungsten ores important for industrial use. For an ideal cubic crystal, the reciprocal elastic modulus can be expressed as $\frac{1}{E}=S_{11}-2\left[\left(S_{11}-S_{12}\right)-\frac{1}{2} S_{44}\right]\left(l^{2} m^{2}+m^{2} n^{2}+l^{2} n^{2}\right)$ where $l$, $m$ and $n$ are the direction cosines of the specimen axis with respect to the crystallographic axis [14]. This is an identical expression to that expressed by Zhang et al.[15] by introducing the $(h, k, l)$ vector $\frac{1}{E(h k l)}=S_{11}-2 S_{A} \frac{h^{2} k^{2}+h^{2} l^{2}+k^{2} l^{2}}{\left(h^{2}+k^{2}+l^{2}\right)}$ with $S_{A}=S_{11}-S_{12}-\frac{1}{2} S_{44}$. Interestingly, for tungsten, the value of $S_{A}$ is close to zero, i.e. the material response of $\mathrm{W}$ is close to that of an isotropic material.

Due to its inert nature, $W$ has been proposed as a candidate material to be used for plasma facing, as a part of structural components required for applications like limiters, first wall armour and divertor components in nuclear fusion reactors [16] and in military applications such as reinforcing materials in alloyed composites for making bullet shot and radiation shields [17]. Furthermore, due to the importance of tungsten in the carbide tooling industry, British Geological Surveys, US Department of Defense and European Commission (EC) have identified tungsten as a 'critical' raw material due to its supply risk. 
Pre-print (Computational Materials Science) - accepted in April 2018

Tungsten is a BCC transition metal, and such metals commonly shear in $<111>$ directions on the (110), (112) and (123) planes [18] and/or sometimes these deformations resemble "pencil glide" on arbitrary planes along the $<111>$ direction whereas $<100>(100)$ was recognized as easy cleavage crystallographic directions. Bahr et al.[19] also observed that when the shear stress prior to yield is slightly higher than the flow stress, a phenomenon referred to as "staircase yielding" prevails.

Noticeably, identical values of shear strength for tungsten $(18 \mathrm{GPa}=0.11 \mathrm{G})$ with failure strains of about $17-18 \%$ in the $<111>$ direction were obtained regardless of the slip plane whereas the ideal tensile strength of the (010) surface obtained was $29 \mathrm{GPa}$. The ideal shear stress (18 GPa) found by Roundy et al. [18] was calculated using a Hertzian solution for the stress field of an elastic indenter, and is considerably lower than the value obtained by Bahr et al.[19], who reported a maximum shear stress for tungsten (oxidized for $32 \mathrm{~h}$ ) of $28.6 \mathrm{GPa}$, and gave an estimation for the shear stress required for dislocation nucleation in pure $\mathrm{W}$ of $26 \mathrm{GPa}$. This difference was attributed to the linear elastic assumption which is used to calculate the values from the experimental data. Consequently, a correction factor in the form of sinusoidal stress-strain relations (to the experimental data by a factor of $2 / \pi$ ) was proposed to eliminate this difference [18]. A summary of these results is shown in table I.

Table I: Experimental data on nanoindentation of tungsten [18]

\begin{tabular}{|c|c|c|}
\hline Maximum normal stress $\left(\sigma_{\max }\right)$ & Maximum shear stress $\left(\tau_{\max }\right)$ & Normalized shear stress $\left(\tau_{\max } / \mathrm{G}\right)$ \\
\hline $69.6 \mathrm{GPa}$ & $21.6 \mathrm{GPa}$ & $13.5(\%)$ \\
\hline
\end{tabular}

In another study, Lassner et al.[20] asserted that slip is the basic mechanism in the plastic deformation of single crystal tungsten, and it occurs in the most densely packed $<111>$ direction on (110) or (122) planes, and additionally on the (111) plane at elevated temperatures. They also noted that twinning is a less dominant deformation mechanism and occurs mainly on the (112) plane in the $<111>$ direction. Argon et al. [21] examined low temperature deformation of single crystal tungsten (in the range of 
Pre-print (Computational Materials Science) - accepted in April 2018

$77 \mathrm{~K}$ to $450 \mathrm{~K}$ ) and concluded that it exhibits an anisotropy on the (112) plane producing slip on unexpected slip systems.

Simulations on BCC metals is an emerging area of research, since little is known about their deformation behaviour at the nanoscale $[22,23]$. This was the major motivation for choosing tungsten as the reference material for this work while comparing different indenters.

\section{Molecular dynamics (MD) simulation of nanoindentation}

\subsection{Simulation setup}

In this work, the "Large-scale Atomic/Molecular Massively Parallel Simulator" (LAMMPS $17^{\text {th }}$ November 2016 version) [24] was used to perform a series of MD simulations. OVITO [25] was used to visualize and analyse the atomistic simulation data while an automated "dislocation extraction algorithm" (DXA) [26] and crystal analysis tool (CAT) [27] were used for automated identification of crystal defects, dislocation lines and their Burgers Vector from the output of the MD data. The algorithm and the necessary considerations required and used in this work are quite similar to that of simulating nanometric cutting described in detail elsewhere $[28,29]$ and are not repeated here for brevity. The model developed in this work assumes periodic boundary conditions along the $X$ and $Z$ directions (Figure 1). The indenter shape was made to closely approximate a $3 \mathrm{~nm}$ radius sphere. In this work, positions of the atoms in the indenter were kept fixed but the boundary condition of the indenter was changed during various simulations. 
Pre-print (Computational Materials Science) - accepted in April 2018

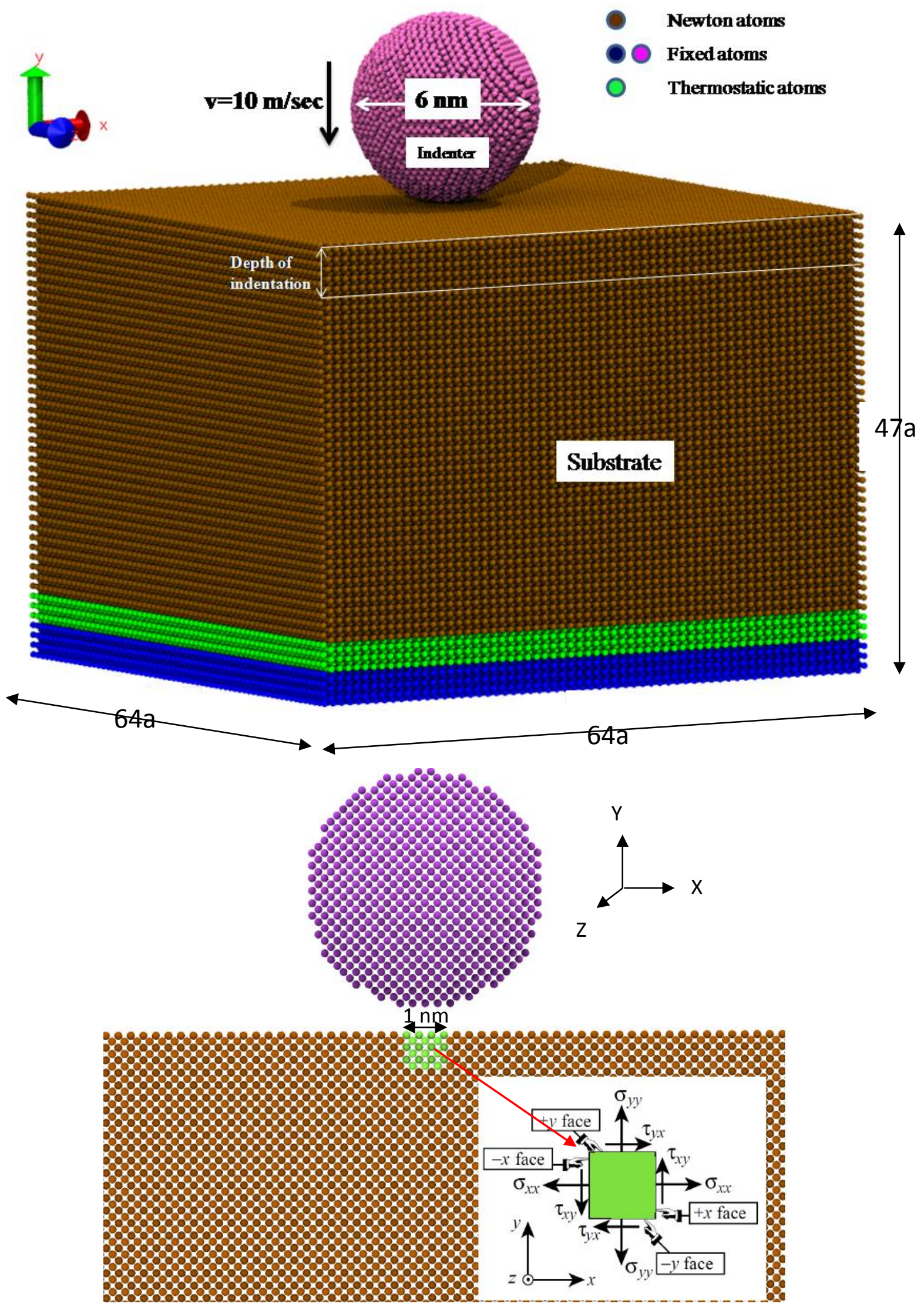

Figure 1: Schematic diagram (a) nanoindentation simulation model with lattice constant (a) = $3.16496 \AA$ (b) volume of material $(1 \mathrm{~nm} \times 1 \mathrm{~nm} \times 1 \mathrm{~nm})$ considered for stress computation (only 2D representation of $3 \mathrm{D}$ volume in $\mathrm{XY}$ plane is shown here) 
Pre-print (Computational Materials Science) - accepted in April 2018

It may be noted that the atomic cohesion in tungsten is controlled by d-band filling. Groger et al.[30] pointed out that the d-electron mediated angular bonding leads to atomic interactions that are noncentral, thereby limiting the applicability of pair-potentials like the Morse or Lennard Jones potentials. In addition, a potential function is usually parametrised for a fixed and known (albeit wide ranging) combination of coordination and bonding topology. This parametrisation strategy for bulk fitting inevitably introduces potential energy barriers (e.g., the Albe barrier), thereby limiting the potential to accurately describe near-surface phenomena: a trade-off between the strain energy and number of dangling bonds leads to a non-physical handling of bond-bending forces. A researcher therefore must be aware that an underestimation of either the strain energy or the number of dangling bonds may lead to an observed non-physical behaviour in the scenario of contact mechanics. This problem is well known in the MD community and led Tersoff to develop separate parametrisations to study surface and bulk silicon [31, 32]. In the many-body potential [33] tested in this study, we notice such potential inconsistencies while indenting $W$ with diamond and tungsten indenters, resulting in very little cohesive force during approach. While this behaviour is not realistic vis-a-vis experiments, we suggest that these inadequacies do not affect deeper plastic events observed subsequently during the indentation. Our claim rests on the Oliver-Pharr $(O \& P)$ analysis applied to the load-displacement $(P-h)$ plots from the simulations, as well as the dislocation behaviour, which together suggest that the reported results in this work are independent of this contact. The details of the various parameters in developing the MD simulation model reported in this work are shown in Table II (which may be useful to replicate our simulation results).

Table II: Details used for the development of MD simulation model

\begin{tabular}{|c|c|}
\hline Dimensions of the tungsten workpiece & $\begin{array}{l}64 \mathrm{a} \times 47 \mathrm{a} \times 64 \mathrm{a} \text { where ' } \mathrm{a} \text { ' is } 3.16496 \AA \\
\text { (equilibrium lattice constant of tungsten at } 10 \mathrm{~K} \text { ) }\end{array}$ \\
\hline $\begin{array}{l}\text { Distance between indenter and specimen } \\
\text { surface }\end{array}$ & $0.5 \mathrm{~nm}$ \\
\hline Depth of indentation & $2 \mathrm{~nm}$ \\
\hline Speed of indentation and retraction & $10 \mathrm{~m} / \mathrm{s}=0.01 \mathrm{~nm} / \mathrm{ps}$ \\
\hline
\end{tabular}


Pre-print (Computational Materials Science) - accepted in April 2018

\begin{tabular}{|l|c|}
\hline Total time (Indentation+retraction) & $(2+0.5 \mathrm{~nm}) / 0.01 \mathrm{~nm} / \mathrm{ps} \times 2=250 \mathrm{ps} \times 2=500 \mathrm{ps}$ \\
\hline Timestep for each calculation & $2 \mathrm{fs}=0.002 \mathrm{ps}$ \\
\hline Total run timesteps & $500 / 0.002=250,000$ \\
\hline Boundary conditions & Periodic in X and Z direction \\
\hline Workpiece crystal orientation & $(010)$ \\
\hline Ensemble used in the simulation & NVE at $10 \mathrm{~K}$ \\
\hline \multicolumn{2}{|c|}{ Simulation test cases: }
\end{tabular}

\section{Case 1: DI}

(i) Spherical shaped rigid diamond indenter (carbon atoms) of radius $\mathrm{R}=3 \mathrm{~nm}$ described by $A B O P$ potential function with both adhesive and repulsive interactions between the indenter and the substrate (RIGID DI- FULL)

(ii) Spherical shaped deformable diamond indenter (carbon atoms) of radius $\mathrm{R}=3 \mathrm{~nm}$ described by ABOP potential function with both adhesive and repulsive interactions between the indenter and the substrate (Deformable DI)

(iii) Spherical shaped rigid diamond indenter (carbon atoms) of radius $\mathrm{R}=3 \mathrm{~nm}$ described by $A B O P$ potential function by manually switching off the pairlike attraction between the $\mathrm{W}-\mathrm{C}$ pair in the original formulation of the ABOP potential to mimic a RI indenter but with realistic repulsive forces (DI-NA).

\section{Case 2: TI}

(iv) Spherical shaped rigid tungsten indenter (tungsten atoms) of radius $\mathrm{R}=3 \mathrm{~nm}$ described by the ABOP potential function (TI)

\section{Case 3: RI}

(v) An imaginary spherical shapedrigid indenter of radius $\mathrm{R}=3 \mathrm{~nm}$ described by a potential with a repulsive force constant $\mathrm{F}(\mathrm{r})=-\mathrm{K}(\mathrm{r}-\mathrm{R})^{2}$ where $\mathrm{K}$ is $2 \mathrm{keV} / \AA^{3}=3204 \mathrm{nN} / \AA^{2}=$ $0.3204 \mathrm{GPa}(\mathbf{R I})$

A low temperature of $10 \mathrm{~K}$ was used to equilibrate the sample and to perform the nanoindentation to avoid potentially confounding effects of thermal fluctuations and thermal vibrations on the final results. Experimentally, it is known that tungsten may react with oxygen to form $\mathrm{WO}_{3}$ at a temperature of about $673 \mathrm{~K}$ and $\mathrm{WO}_{3}$ sublimes further at about $1073 \mathrm{~K}$. In general, experimental conditions often involve the presence of oxide layers which create a passivation layer over the indenting surface. To mimic these conditions, Kelchner et al.[11] introduced an imaginary spherical repulsive rigid indenter 
Pre-print (Computational Materials Science) - accepted in April 2018

(RI) with a force potential. Considering these conditions, we chose the following five cases to understand the influence of the indenter:

(i) A rigid diamond indenter of radius $\mathrm{R}=3 \mathrm{~nm}$ fully described by the ABOP potential function with both adhesive and repulsive interactions between the diamond indenter and the tungsten substrate (RIGID DI- FULL)

(ii) A deformable diamond indenter of radius $\mathrm{R}=3 \mathrm{~nm}$ described by the $\mathrm{ABOP}$ potential function with both adhesive and repulsive interactions between the diamond indenter and the tungsten substrate (Deformable DI)

(iii) An imaginary spherical rigid indenter of radius $\mathrm{R}=3 \mathrm{~nm}$ described by a hypothetical repulsive force potential with a force constant $F(r)=-K(r-R)^{2}$ where $K$ is $2 \mathrm{keV} / \AA^{3}=3204$ $\mathrm{nN} / \AA^{2}=0.3204 \mathrm{GPa}(\mathbf{R I})$

(iv)A rigid but modified diamond indenter of radius $\mathrm{R}=3 \mathrm{~nm}$ described by the ABOP potential function with modifications made in the potential interactions by manually switching off the pair-like attraction between the $\mathrm{W}-\mathrm{C}$ pair in the original formulation of the ABOP potential to mimic the RI indenter by accounting for the realistic repulsive forces (DI-NA) i.e. by setting the value of $V_{A}=0$ in the ABOP potential function [33]

(v) A rigid tungsten indenter (tungsten atoms) of radius $R=3 \mathrm{~nm}$ described by the ABOP potential function (TI)

Based on these five test cases, the nanoindentation results were analyzed with respect to the contact pressure $\left(p_{m}\right)$ underneath the indenter. Contact pressure $\left(p_{m}\right)$ is defined as the ratio of the instantaneous normal force $(F)$ on the indenter and the projected contact area $(A)$. 

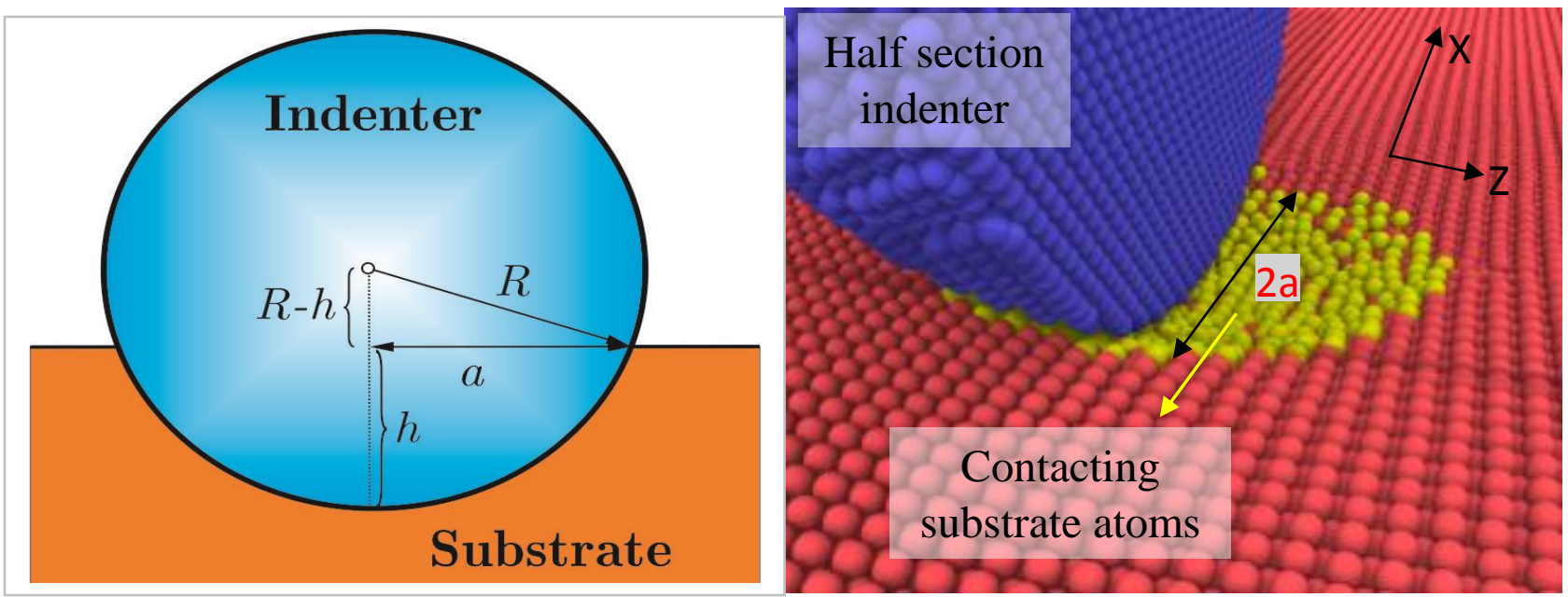

Figure 2: Schematic illustration of the scheme to estimate the projected contact area by using (a) geometry based area function approach i.e. $\left(\pi \times \mathrm{a}^{2}\right)$ where $a=\sqrt{h(2 R-h)}$ is the projected contact radius, so that $R$ is the radius of the indenter and $h$ is the instantaneous displacement of the indenter [34] and (b) screening the contact atoms using fixed cut off as per the ABOP potential function and then using $\left(\pi \times \mathrm{a}^{2}\right)$ formulation where $2 a$ is the contact diameter taken as the largest distance determined from the $\left(X_{\text {high }}-X_{\text {low }}\right)$ and $\left(Z_{\text {high }}-Z_{\text {low }}\right)$ values with $X$ and $Z$ being the atomic coordinates

Building on recent practice $[1,35]$, we constructed the contact area by determining the size of the contiguous region of the $W$ sample atoms involved in a chemical interaction with the indenter at a given instance. We took a cutoff distance (defined by the potential function) from the indenter to count the number of atoms in the substrate that are in contact with the indenter. The cut off distances as per the ABOP potential are $3.0 \AA$ (Carbon-Tungsten) and $3.8 \AA$ (Tungsten-Tungsten) respectively. This in turn gives us a 3-dimensional elliptical contact area underneath the indenter as shown by the yellow atoms highlighted in figure 2 (b).

After mapping the number of contacts, we evaluated the $X$ and $Z$ coordinates and took the largest of these to obtain the projected contact diameter $2 a$, and hence $\pi a^{2}$ the chemical contact area. We compared this atomistic projected contact area against the purely continuum geometric approach of the Oliver and Pharr method [34] where the projected contact area for a spherical indenter is expressed as $\left(\pi \times \mathrm{a}^{2}\right)$ (here $\mathrm{a}=\sqrt{h(2 R-h)}$ is the contact radius of the spherical indenter, $R$ is the radius of the indenter and $h$ is the instantaneous displacement of the indenter) [36]. Interestingly, in the geometric estimation approach, the contact area remains unchanged with the indenter type, especially when the 
Pre-print (Computational Materials Science) - accepted in April 2018

indentation force changes with the change of the atomic species in the indenter. In the chemical approach proposed in this work, both the contact area as well as the indentation force change with the change in the material and the indenter's boundary condition, and this helps increase the accuracy of the estimation. For a given indenter shape, the projected area of a residual impression (i.e., plastic deformation) divided by the peak indentation load used to form it is known as the hardness $H$ of the material (for that shape). The contact pressure $\left(p_{m}\right)$ or true stress can be plotted against true strain during the nanoindentation [37] to calculate the elastic modulus of the material and determine the elastic-plastic limit of the substrate material. An alternative method for calculating the hardness from a spherical indenter in the MD simulation framework also exists [38] but is only applicable to a rigid indenter, while our proposed method is applicable for any indenter type. We compared the results of the projected contact area and hardness obtained through our method with that of the geometric estimations (these are presented and discussed later).

In addition to hardness, nanoindentation is also used to characterise the elasticity of the material. The most popular way of calculating elastic modulus $(E)$ of the material from the load-displacement $(P$ h) plot was proposed by Oliver and Pharr $(O \& P)$ [34]. The method relies on the calculation of the projected contact area from a power-law fitted to the unloading curve of the $P$ - $h$ plot. The slope of this curve $(S)$ enables one to obtain the reduced elastic modulus of the material which can then be used to obtain $E$ using the following equations:

$\frac{1-\vartheta^{2}}{E}=\frac{1}{E_{r}}-\frac{1-\vartheta_{\text {indenter }}^{2}}{E_{\text {indenter }}}$

where $E_{r}$ is the reduced modulus while $v$ is the Poisson's ratio.

For our work, where the indenter was modelled as a fully rigid body, the value of $E_{\text {indenter }}$ is infinite; thus, the equation simplifies to:

$E=E_{r} \times\left(1-\vartheta^{2}\right)$ 
Pre-print (Computational Materials Science) - accepted in April 2018

where the value of $v$ (Poisson's ratio of tungsten) was taken as 0.27 as calculated from an MD simulation of test for elastic constant using the ABOP potential function shown later in Table III.

$E_{r}$ can be obtained by the following equation:

$E_{r}=\frac{1}{\beta} \frac{\sqrt{\pi}}{2} \frac{S}{\sqrt{A}}$

where $S$ is the slope of the top $1 / 3^{\text {rd }}$ part of the unloading curve which were obtained as $2392 \mathrm{~N} / \mathrm{m}$ (for DI), $3496 \mathrm{~N} / \mathrm{m}$ (for $\mathrm{TI}$ ) and $1715 \mathrm{~N} / \mathrm{m}$ (for RI) respectively from the simulation results at an indentation depth $2 \mathrm{~nm}, \beta=1$ is a constant for spherical indenter and $A$ is the projected area $\left(\mathrm{m}^{2}\right)$ which varies with the depth of indentation.

\subsection{Comparison of potential energy functions}

Prior to carrying out the nanoindentation simulations in this work, three potential functions available in the literature were tested for their accuracy in reproducing the elastic constants and other important mechanical properties such as Zener anisotropy ratio, Young's modulus, shear modulus, Voigt Poisson's ratio and Voigt Bulk modulus of tungsten. These values are shown and compared with results from experiments in table III for reference. It can be seen that the Zener anisotropic ratio for tungsten is close to unity and that all three potential functions MEAM [39], ABOP [33] and Finnis Sinclair type EAM [7, 40] proposed for tungsten reproduce the mechanical properties reasonably well. In the past, one of the authors of this work compared five different potentials and studied screw dislocation mobilities on the (112) glide plane in $W$ [39]. In that study, the modified embedded-atom potential was shown to have the best compromise in terms of static and dynamic screw dislocation properties. However, since ABOP has been parametrised fully for W-C interactions it became an obvious choice for this work. Figure 3 shows the dimer energy (having fitting characteristics like the Rydberg potential energy curve) and tensile force of the dimer for different pairs like (C-C), (W-W), (W-C) and (W-purely repulsive) obtained from ABOP [33]. It may be noted that the ABOP potential, in spite of being a many-body potential, is a short-range potential function employing a cut-off that uses trigonometric functions. The scheme employed in the development of this empirical bond-order 
Pre-print (Computational Materials Science) - accepted in April 2018

potential function assumes perfect screening for second and farther neighbours and sets their bond integrals to zero. This assumption leads the dimer energy to drop to zero steeply as the cut-off is approached and it has already been alluded to as a bottleneck [41]. Despite this non-physical behaviour (which may be corrected by employing a refined screening function-based approach), the basic differences in the dimer energy and forces between the kind of pairs involved in the study i.e. $\mathrm{C}-\mathrm{C}, \mathrm{W}-\mathrm{W}, \mathrm{W}-\mathrm{C}$ and $\mathrm{W}$-purely repulsive are evident from figure 3 . It is striking that with the involvement of a purely repulsive indenter described by a force potential, there is no dimer energy involved but only a force that is experienced by the contacting atom within a fraction of few Angstroms. The obvious difference in the dimer energy between a W-Purely repulsive interaction and the $\mathrm{W}-\mathrm{C}$ interaction provides a microscopic picture of the expected differences between the two indenters, namely DI and RI, which are discussed further in rest of the sections.
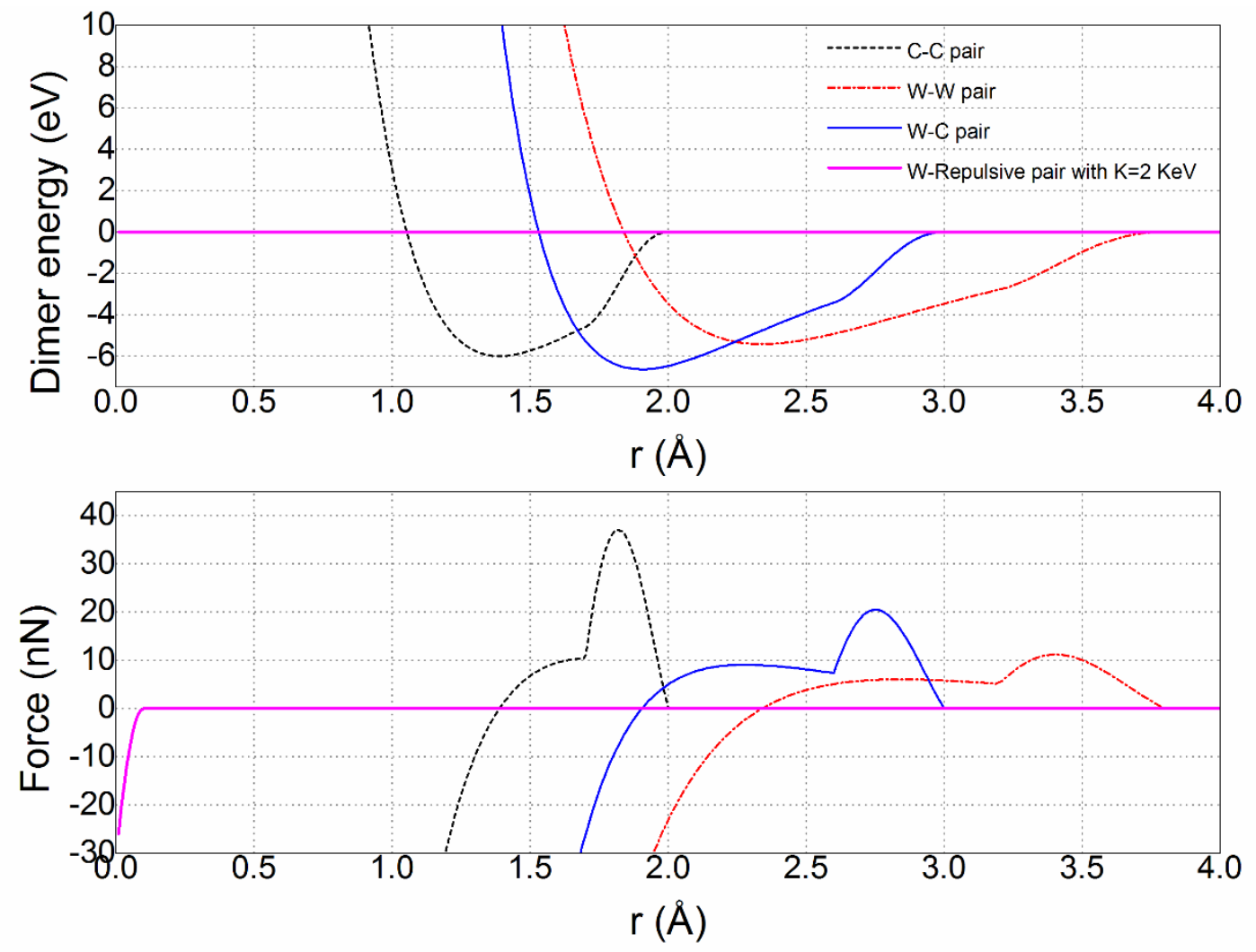

Figure 3: Dimer energy exhibiting characteristic of the Rydberg potential energy curve and pairforces as a function of distance $r$ between the asperity moving towards a tungsten atom 
Pre-print (Computational Materials Science) - accepted in April 2018

Table III: Experimental properties of tungsten compared with the values obtained from the MD simulation at $0 \mathrm{~K}$ using different types of potential energy functions

\begin{tabular}{|c|c|c|c|c|}
\hline Properties of tungsten & $\begin{array}{l}\text { Spline based } \\
\text { MEAM [39] }\end{array}$ & $\begin{array}{c}\text { ABOP } \\
{[33]}\end{array}$ & $\begin{array}{l}\text { Finnis-Sinclair } \\
\text { (EAM) }[40]\end{array}$ & $\begin{array}{c}\text { Experimental } \\
\text { values }^{1}[42]\end{array}$ \\
\hline Equilibrium lattice constant $(\AA)$ & 3.188193 & 3.16496 & 3.1652 & 3.155 \\
\hline$C_{11}=C_{22}=C_{33}(\mathrm{GPa})$ & 523.99 & 541.775 & 532.612 & 501 \\
\hline $\mathrm{C}_{12}=\mathrm{C}_{13}=\mathrm{C}_{23}(\mathrm{GPa})$ & 204.916 & $\begin{array}{c}191.023 \\
6\end{array}$ & 205.016 & 198 \\
\hline $\mathrm{C}_{44}=\mathrm{C}_{55}=\mathrm{C}_{66}(\mathrm{GPa})$ & 161.223 & $\begin{array}{c}161.919 \\
7\end{array}$ & 163.198 & 151.4 \\
\hline Zener anisotropy ratio $\frac{C_{11}-C_{12}}{2 \times C_{44}}$ & 0.9895 & 1.08 & 1 & 1 \\
\hline $\begin{array}{l}\text { Young's modulus }\left(\mathrm{E}_{100}\right)[43](\mathrm{GPa}) \\
C_{11}-2 \frac{C_{12}}{C_{11}+C_{12}} C_{12}\end{array}$ & 408.7747 & $\begin{array}{c}442.184 \\
2\end{array}$ & 418.65 & 388.828 \\
\hline $\begin{array}{l}\text { Young's modulus }\left(\mathrm{E}_{110}\right)[43](\mathrm{GPa}) \\
4 \frac{\left(C_{11}{ }^{2}+C_{12} C_{11}-2 C_{12}{ }^{2}\right) C_{44}}{2 C_{44} C_{11}+C_{11}{ }^{2}+C_{12} C_{11}-2 C_{12}^{2}}\end{array}$ & 411.531 & 420.174 & 417.67 & 388.664 \\
\hline $\begin{array}{l}\text { Young's modulus }\left(\mathrm{E}_{111}\right)[43](\mathrm{GPa}) \\
3 \frac{C_{44}\left(C_{11}+2 C_{12}\right)}{C_{11}+2 C_{12}+C_{44}}\end{array}$ & 412.4586 & $\begin{array}{c}413.316 \\
5\end{array}$ & 417.34 & 388.6087 \\
\hline $\begin{array}{l}\text { Shear modulus }(\mathrm{G})[44](\mathrm{GPa}) \\
\frac{C_{11}-C_{12}+C_{44}}{3}\end{array}$ & 160.099 & $\begin{array}{c}170.890 \\
4\end{array}$ & 151.47 & 151.4667 \\
\hline $\begin{array}{l}\text { Voigt Poisson's ratio [44] } \\
\frac{C_{11}+4 C_{12}-2 C_{44}}{4 C_{11}+6 C_{12}+2 C_{44}}\end{array}$ & 0.2799 & 0.27 & 0.27836 & 0.2833 \\
\hline $\begin{array}{l}\text { Voigt Bulk Modulus(B) (GPa) [45] } \\
\frac{\left(C_{11}+C_{22}+C_{33}\right)+2\left(C_{12}+C_{13}+C_{23}\right)}{9} \\
\text { or } \frac{\left(C_{11}+2 C_{12}\right)}{3} \text { for isotropic }\end{array}$ & 311.274 & 307.941 & 314.215 & 299 \\
\hline
\end{tabular}

\footnotetext{
${ }^{1}$ Experimentally E of tungsten is considered in the range of 409 to $413 \mathrm{GPa}$
} 
Pre-print (Computational Materials Science) - accepted in April 2018

\subsection{Results and discussions}

\subsection{P-h plots and incipient plasticity}

The $P$ - $h$ plots obtained from the MD simulation for all five test cases: (i) RIGID DI - FULL, (ii) Deformable DI, (iii) Rigid DI-NA, (iv) TI and (v) RIfor with the simulations performed using the same parameters at a temperature of $\mathbf{1 0} \mathbf{K}$, are shown and compared in figure 4 . In these plots, it may be noted that the rigid indenter travelled $0.5 \mathrm{~nm}$ to reach the (relaxed) surface level of the specimen and then advanced by a further $2 \mathrm{~nm}$ so that it achieved a total of $2.5 \mathrm{~nm}$ displacement with a velocity of $10 \mathrm{~m} / \mathrm{s}$. The exact position of the contact depended on substrate relaxation under tip-sample interactions.

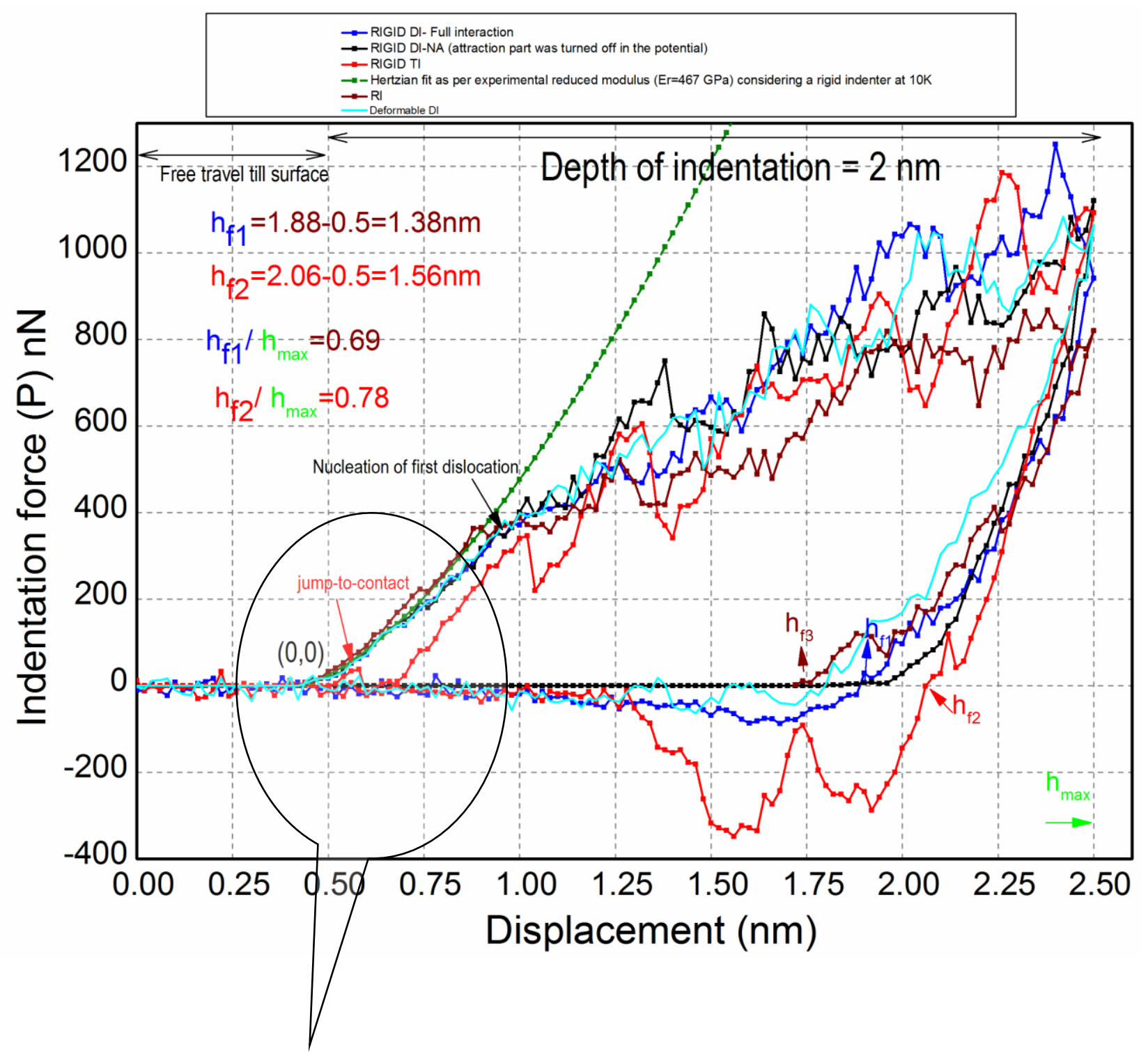


Pre-print (Computational Materials Science) - accepted in April 2018
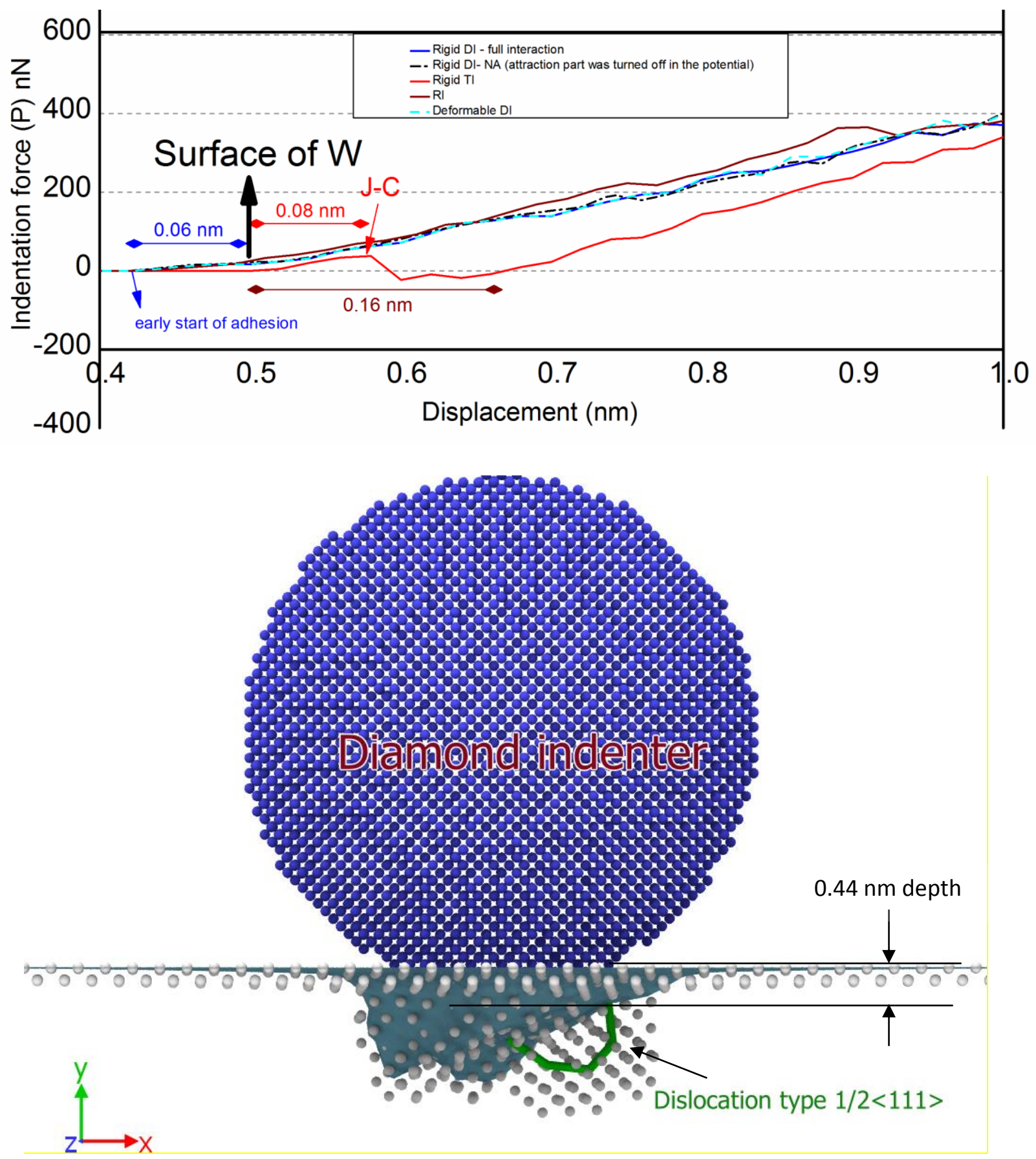

Figure 4: (a) P-h plots obtained from the MD simulation for the fivedifferent type of indenters simulated for the nanoindentation of $W$ at $10 \mathbf{K}$. The dashed green line is a fit of the initial elastic loading section to the Hertzian contact law ( Force $=4 / 3 E_{r} R^{1 / 2} h^{3 / 2}$ ) (Experimental value of $E_{r}$ and $E$ for $W$ pressed with diamond are known as $~ 323 \mathrm{GPa}$ and $429 \mathrm{GPa}$ (at $300 \mathrm{~K}$ ) respectively [46]. Accounting for a rigid indenter and considering $1 \%$ temperature increase from $300 \mathrm{~K}$ to $10 \mathrm{~K}$ for an imaginary experimental rigid indenter at $10 \mathrm{~K}$ provides an $E_{r}$ of $467 \mathrm{GPa}$ ) (b) A close up view of the different types of indenters approaching the substrate surface are also shown to highlight the near surface phenomena such as Jump-to-contact observed only with TI(c) First dislocation of type $\mathbf{b}=1 / 2$ $<111>$ nucleated at an indentation depth of $0.44 \mathrm{~nm}$ as highlighted in the P-h plot 
Pre-print (Computational Materials Science) - accepted in April 2018

Note that in the O\&P equation [34], we made the value of the elastic modulus of diamond as infinite and obtained the experimental value of $E_{r}$ for a rigid indenter as $467 \mathrm{GPa}$. This value of $E_{r}$ was used as an input for plotting the Hertz elastic contact behavior shown in the green dashed line in Figure 4a, using the equation Force $=4 / 3 E_{r} R^{1 / 2} h^{3 / 2}$. Also, the RI response in Figure 4a was offset by $(-0.25$ $\mathrm{nm})$ to show the congruent overlay more clearly.

In what follows, we present a detailed phenomenology of the $P$ - $h$ behaviour of each indenter type (figure 4a). Upon contact, the RI exhibited an apparent elastic load-up regime with a slight noise ("atomistic chatter"). This compares well with a continuum elastic $3 \mathrm{~nm}$ Hertzian load-up. At about $0.94 \mathrm{~nm}$ (corresponding to an indentation depth of $0.44 \mathrm{~nm}$ ), a sharp slope change occurs in a region of higher chatter. In the MD, this is the point of first inelastic behaviour. A magnified observation (shown in figure $4 c$ ) reveals that the first dislocation of type $b=1 / 2<111>$ nucleated at this point (at an indentation depth of $0.44 \mathrm{~nm}$ ). At about $2.25 \mathrm{~nm}$, the load peaks and an extended drop in the $P$ - $h$ behaviour ensues to the final displacement. The unload region displays a smooth sharp drop, characteristic of elastic unloading. A careful review of the atomic behaviour in this unloading regime reveals no further sub-surface inelastic behaviour with minor atomic rearrangements at the periphery of the indenter-substrate interface. We analyse the reduced elastic modulus later in the paper using O\&P. As a next step, a rigid diamond indenter was added into this comparison with the adhesive part of the interaction switched off (DI-NA). This was done by setting $\mathrm{V}_{\mathrm{A}}=0$ in the $\mathrm{ABOP}$ potential function i.e. the pairlike attraction energy between the W-C pair in the original formulation of the ABOP potential was made equal to 0 [33] to emulate a more realistic repulsive indenter. The initial load-up is within the bounds of small chatter, virtually identical to that of the RI. At about $1 \mathrm{~nm}$, a sharp slope reduction occurs initially, which is quite similar to that of the RI. However, subsequent displacement beyond $0.5 \mathrm{~nm}$ led the DI-NA to take on a significantly different character exhibiting large undulations with a mean load that is about $25 \%$ higher than that of the RI. The undulations have a peak-to-peak difference of up to $200 \mathrm{nN}$ and a rough period of 0.5 to $0.6 \mathrm{~nm}$, in stark contrast to the RI chatter in this regime (of $\sim 50 \mathrm{nN}$ ). The atomic corrugation (atomic level roughness) of the tip 
Pre-print (Computational Materials Science) - accepted in April 2018

apparently induces this difference by itself. One way of tackling this problem of surface roughness induced effects has recently been suggested [47]. Upon unloading at $2.5 \mathrm{~nm}$, the DI-NA curve shows a sharp, smooth drop in load with slope very similar to that of the RI. In Figure 4a, the DI- full interactions showed smooth initial load up, congruent with both the RI and DI-NA, with initial divergence from the Hertzian curve similar to these indenters as well at $1 \mathrm{~nm}$. However, beyond this, the influence of the adhesion interaction appears strongly as large but distinctly incongruent undulation with the DI-NA. At unload, a largely similar steep curve occurs, primarily differing because of the presence of a small but distinct, low chatter pull off adhesion peaked at about $100 \mathrm{nN}$. This pull-off curve extends back to about $0.7 \mathrm{~nm}$ before the indenter separates from the substrate.

The TI indenter of $3 \mathrm{~nm}$ nominal radius formed from a $W$ lattice with full adhesive and repulsive interaction is also represented in figure $4 \mathrm{a}$. Here the effect of adhesion is revealed as a further distinct perturbation of the Hertzian contact. After an initial small load up to $50 \mathrm{nN}$, a sudden load drop occurs to approximately $0.7 \mathrm{~nm}$ that is associated with a jump-to-contact of substrate atoms with tip atoms one layer back from the apex layer. A sharper load-up over $\sim 0.5 \mathrm{~nm}$ occurs before another jump-to contact load drop from $350 \mathrm{nN}$ to $220 \mathrm{nN}$, which appears just beyond the divergence from the Hertzian curve. The $P$ - $h$ curve for the TI then proceeds with distinct undulations even larger than the DI tips. Upon unload at $2.5 \mathrm{~nm}$, the drop-in load is sharper than for all the other indenters as might be expected from the influence of adhesion. Pull-off behaviour appears much more disturbed by atomic rearrangement with large undulations.

We have presented these phenomenon as progressive effects of atomic corrugation and adhesive interaction on the basic RI response. Of immediate note is the perturbative nature with an underlying conventional indentation behaviour still present. In what follows, we proceed with a standard O\&P analysis.

The plots were compared to highlight some of the salient features such as (i) presence of an adhesive pull-off force of up to $\sim 350 \mathrm{nN}$ between the substrate and the indenter atoms for the TI interaction, 
Pre-print (Computational Materials Science) - accepted in April 2018

(ii) a factor of two spread in peak normal forces on the indenters at a common maximum depth of indentation and (iii) variation in the residual depth of indentation $\left(h_{f} / h_{\max }\right)$ of 0.7 . The residual depth $h_{f}$ signifies the extent of permanent plastic alteration of the substrate during the timescale of the simulation. Note that the slope of the unloading curve and the peak load for the RI significantly differs from that of DI and TI.

We checked the sensitivity of the value of the force constant $K$ in the RI case on the $P$ - $h$ plot, a dummy trial by changing the value of $K$ from $2 \mathrm{keV} / \AA^{3}(0.3204 \mathrm{GPa})$ to $1 \mathrm{keV} / \AA^{3}(0.1602 \mathrm{GPa})$. However, the results reported here remained robust.

In general, the load on the indenter during loading decreases abruptly at certain points, a phenomenon observed experimentally in other atomistic indentation systems [48]. The simulation video shows that this happens due to the release of the accumulated elastic strain as sudden dislocation structures give way under the indenter. A principle notion from continuum elasticity explaining the nucleation of dislocations is that the maximum-resolved shear stress exceeds a critical shear stress value for the indented material. For indentation, this is valid only down to a certain limit: in our case, the size of stressed material requires a very small dislocation loop with large energy cost due to its curvature. At such small scales, a more fundamental criterion suggested for nucleation of plastic deformation is that the work done over the displaced surface should be greater than the line energy of the new dislocation loop [11].

The results shown in figure 4 are further quantified and tabulated in table IV. The peak load in the case of RI was observed to be lower ( $\sim 30 \%$ lower) than that for the DI. Consequently, the hardness computed from the RI was minimal and much lower than that obtained while using the DI and TI. 
Pre-print (Computational Materials Science) - accepted in April 2018

Table IV: Summary of results obtained from the MD simulation $(P-h$ plots $)$

\begin{tabular}{|c|c|c|c|c|}
\hline \multirow{2}{*}{ Type of indenter } & $\begin{array}{c}\text { Peak Load } \\
(\mathbf{n N}) \text { at 2.0 } \mathbf{~ n m} \\
\text { displacement }\end{array}$ & $\mathbf{h}_{\mathbf{f} / \mathbf{h}_{\text {max }}}$ & $\begin{array}{c}\text { Average hardness } \\
\text { (GPa) as per O\&P } \\
\text { geometric method } \\
{[\mathbf{3 4}]}\end{array}$ & $\begin{array}{c}\text { Average hardness } \\
\text { (GPa) as per direct } \\
\text { determination of } \\
\text { interacting region size }\end{array}$ \\
\cline { 3 - 5 } & 1250 & 0.69 & \multicolumn{2}{|c|}{ (for indent depths > 0.6 nm) } \\
\hline DI & 1185 & 0.69 & 32 & 36 \\
\hline TI & 808 & 0.78 & 29 & Not applicable \\
\hline RI & & & & \\
\hline
\end{tabular}

\subsection{Analysis of dislocations and crystal defects by spherical indentation of the tungsten substrate}

Figure 5 shows distinctly different forms of defect structures in the plastic zone at peak indentation depth of $h=2 \mathrm{~nm}$ for each of the three indenter types. Summary details of these defects are provided in table V. Prismatic dislocation loops were found responsible for the irreversible, plastic transport of material in all three cases. Two major types of dislocations with Burgers vectors $\mathbf{b}=1 / 2<111\rangle$ (green colour) and $\mathbf{b}=\langle 100\rangle$ (pink colour) $^{2}$ were revealed. The $\langle 100\rangle$ dislocations were observed to emerge from the interaction between the two $1 / 2<111>$ types. The total number of segments and the total path length of the dislocations generated by the atomically rough, adhesive DI and TI indenters were similar, while the smooth, purely repulsive RI tip showed comparatively smaller lengths of dislocation segments.

\footnotetext{
${ }^{2}$ Readers are requested to refer the web-based version of this article for correct interpretation of the colour legends
} 
Pre-print (Computational Materials Science) - accepted in April 2018

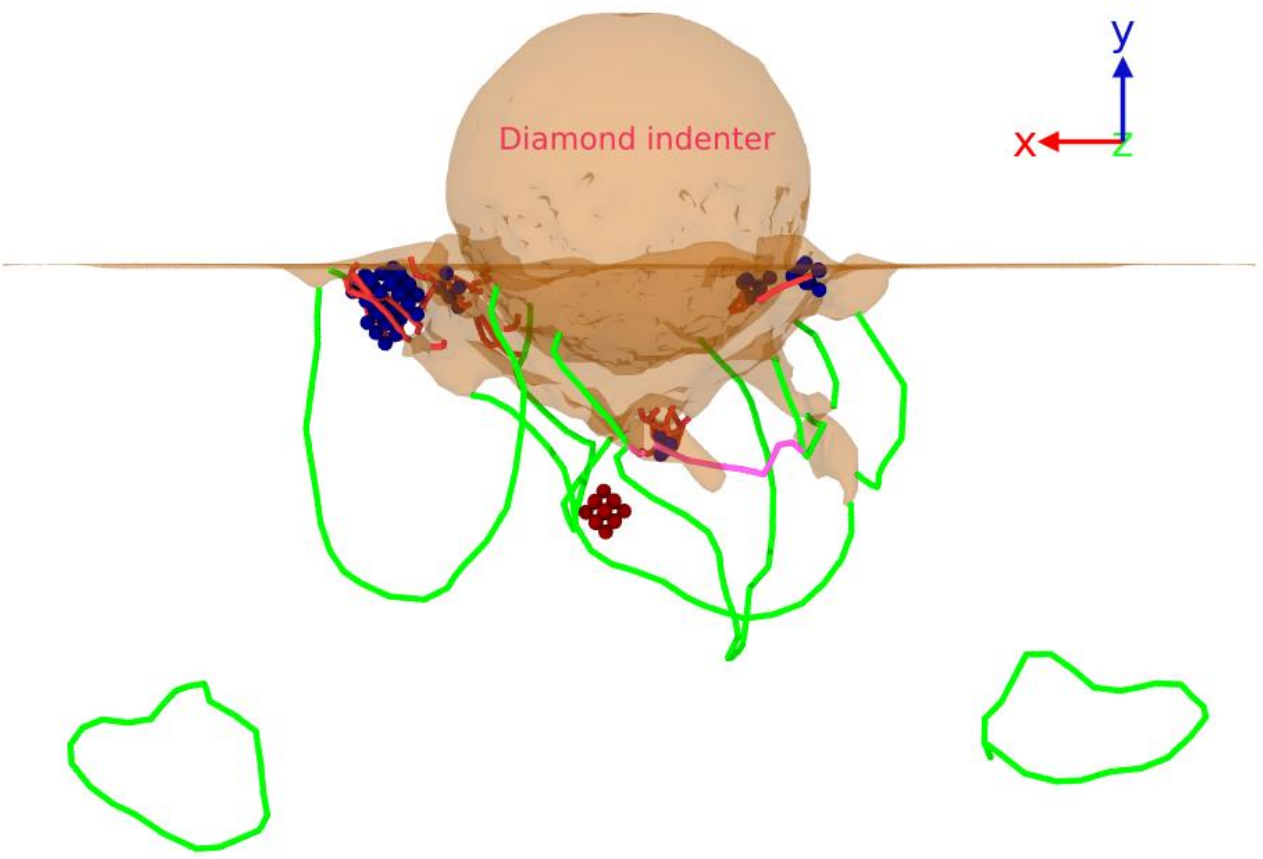

Red clusters are atoms surrounding single BCC vacancy while blue clusters are BCC twin boundaries Length of $b=1 / 2<111>$ type $=84.23 \mathrm{~nm}$, Number of dislocation segments $=13$

Length of $b=<100>$ type $=3.36 \mathrm{~nm}$ Number of dislocation segments $=1$

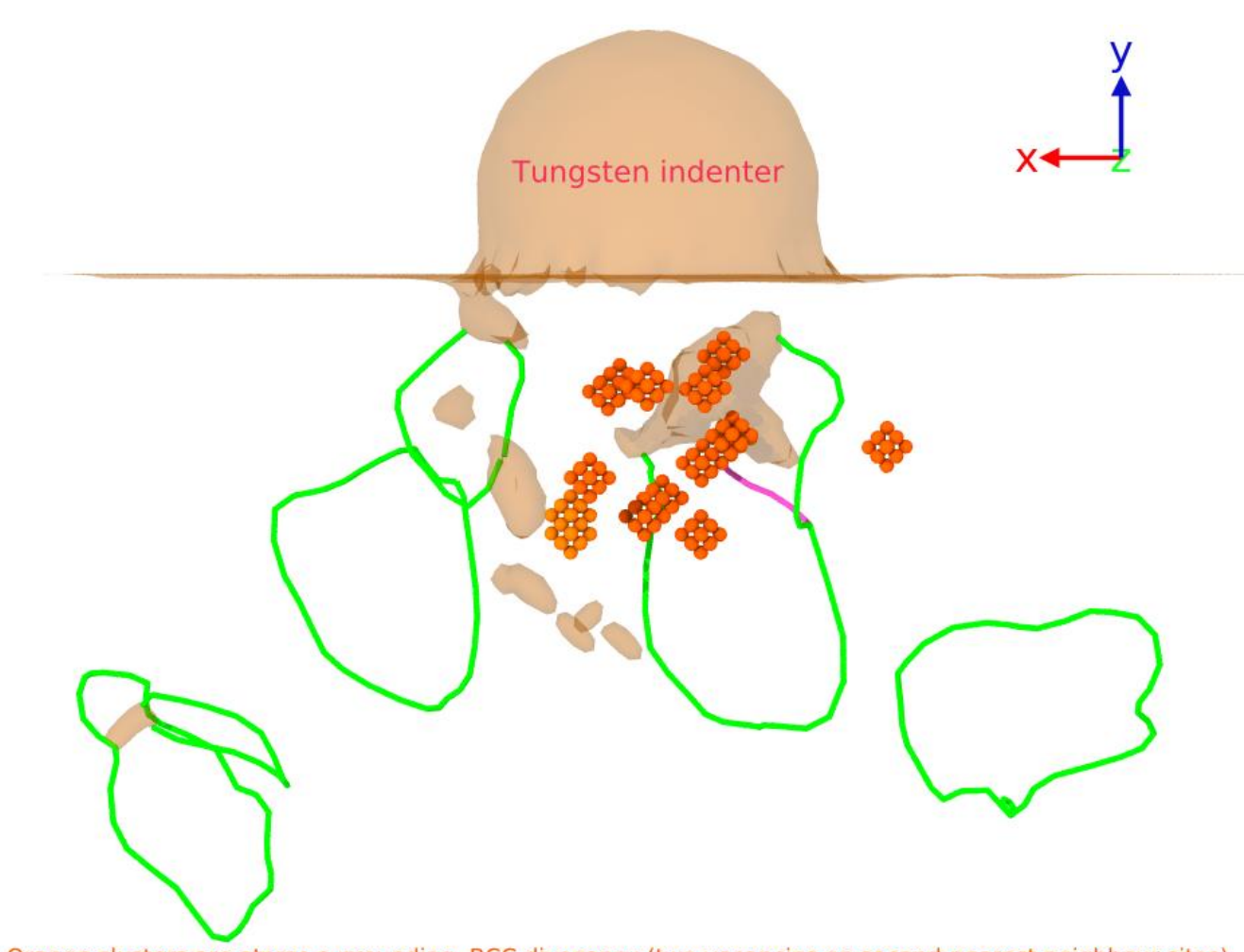

Orange clusters are atoms surrounding BCC divacancy (two vacancies on second-nearest neighbour sites) Length of $b=1 / 2<111>$ type $=85.3 \mathrm{~nm}$, Number of dislocation segments $=10$

Length of $\mathrm{b}=<100>$ type $=3.36 \mathrm{~nm}$ Number of dislocation segments $=2$ 


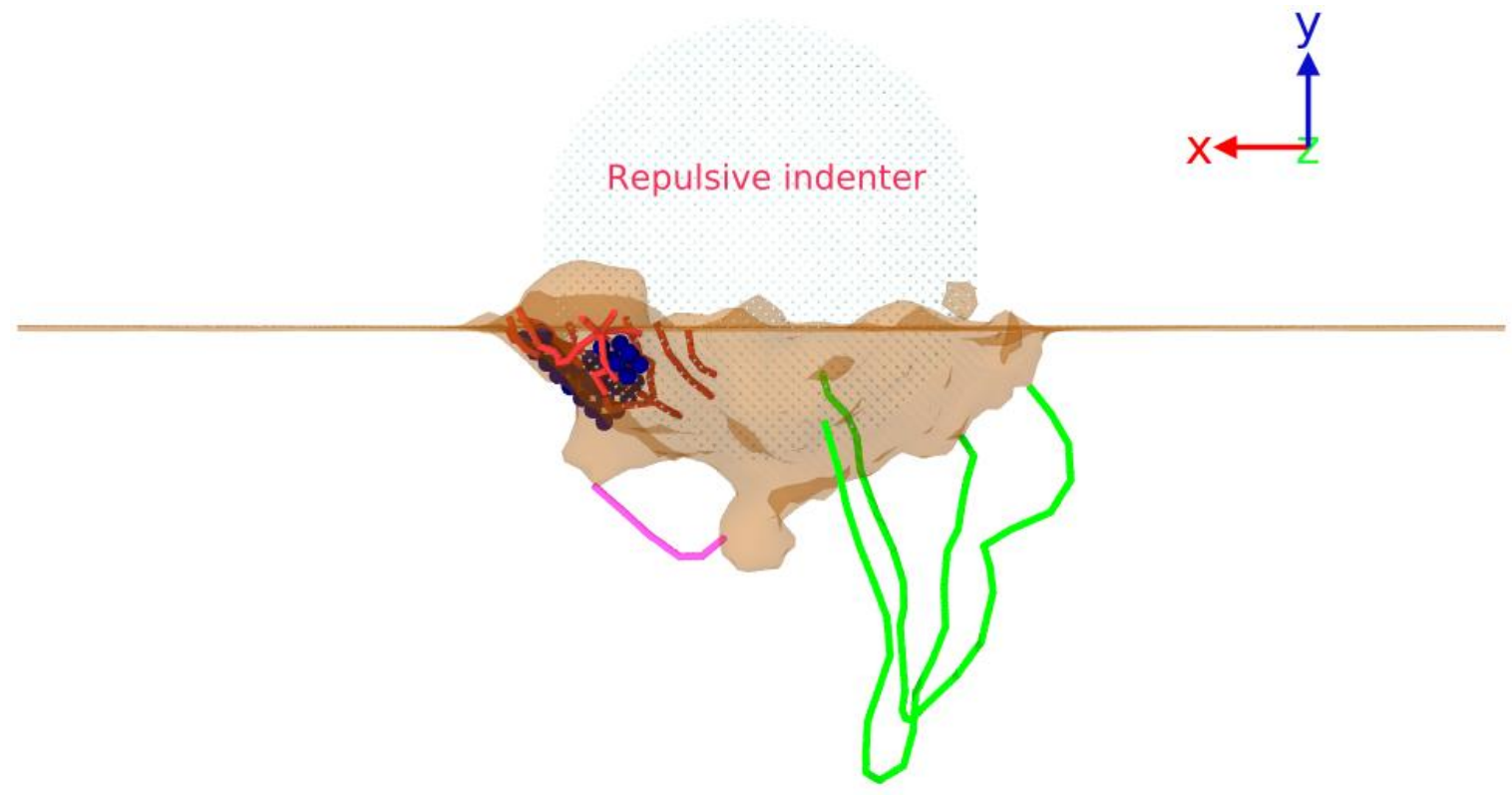

Blue clusters are $\mathrm{BCC}$ twin boundaries

Length of $b=1 / 2<111>$ type $=25.68 \mathrm{~nm}$, Number of dislocation segments $=4$

Length of $b=<100>$ type $=3.36 \mathrm{~nm}$ Number of dislocation segments $=1$

Figure 5: Views in the XY planes obtained from DXA processing shows plastic deformation zone, crystal defects and free surfaces comparing sample defects created for diamond (DI-full), tungsten (TI) and smooth repulsive (RI) at a penetration depth of $2 \mathrm{~nm}$. Dislocations with $\mathbf{b}=1 / 2\langle 111\rangle$ are shown in green and $\mathbf{b}=\langle 100\rangle$ are shown in pink. Common neighbour analysis was used to identify and remove the crystalline atoms to highlight crystalline defects. Red atoms represent atoms surrounding a single BCC vacancy while orange clusters are atoms surrounding a BCC divacancy (two vacancies on second-nearest neighbour sites) and blue atoms represent BCC twin boundaries. The smooth RI indenter is represented for visualisation but it was not physically present in the simulation

To quantify the extent of dislocations, we adopt the notion of volumetric dislocation density $\left(\mathrm{m}^{-2}\right)$, defined as total length of dislocations per unit plastic volume [49] expressed as follows:

Volumetric dislocation density $\left(m^{-2}\right)=\frac{\text { Total leng th of dislocations }}{\left(2 \pi \mathrm{R}_{\mathrm{pl}}^{3} / 3\right)-\left(2 \pi a^{3} / 3\right)}$

where $R_{p l}$ is the radius of the plastic zone (largest distance of a dislocation from the assumed hemispherical indentation point), and for an indentation depth of $h$, the indented volume is also considered hemispherical with projected radius $a$. The average dislocation density induced by $3 \mathrm{~nm}$ radius spherical indentation into pristine, dislocation free $W$ to a $2 \mathrm{~nm}$ depth at $10 \mathrm{~K}$ was found to be in the range $2 \times 10^{16} \mathrm{~m}^{-2}$ to $3 \times 10^{16} \mathrm{~m}^{-2}$. Considering nucleation of dislocation to be of a stochastic 
Pre-print (Computational Materials Science) - accepted in April 2018

nature, a qualitative deduction from these results is that although all three methods show an almost similar extent of dislocation density, the volume of dislocation plasticity (sum of length of all dislocation segments) induced by the RI was somewhat lower than that induced by the DI and TI. Apart from prismatic dislocation loops, some other crystal defects below the indenter were also observed, the most important of which were BCC vacancies and twin boundaries; these are also highlighted in figure 5. The twin boundaries observed appear to be twin embryos in the embryonic stage (very small) and twinning does not seem to contribute to plasticity in these simulations. In figure 5 , red clusters refer to atoms surrounding a single BCC vacancy, the orange clusters refer to $2 \mathrm{NN}$ type BCC divacancy (two vacancies on second-nearest neighbour sites) and the blue coloured atoms refer to twin boundaries. Table $\mathrm{V}$ shows a summary of results highlighting the crystalline defects observed in the three cases.

Table V: Summary of results showing crystalline defects and dislocations type while indenting $W$

\begin{tabular}{|c|c|c|}
\hline Indenter type & Crystalline defect type & Dislocation type \\
\hline DI & $\begin{array}{r}\text { Single BCC vacancy (red) and twin boundaries } \\
\text { (blue atoms) }\end{array}$ & $\mathbf{b}=1 / 2<111>$ and $\mathbf{b}=<100\rangle$ \\
\hline TI & 2 NN type BCC divacancy (orange atoms) & $\mathbf{b}=1 / 2<111>$ and $\mathbf{b}=<100\rangle$ \\
\hline RI & Twin boundaries (blue atoms) & $\mathbf{b}=1 / 2<111>$ and $\mathbf{b}=<100\rangle$ \\
\hline
\end{tabular}

When indented with the DI, two types of defects, namely single BCC vacancy and twin boundaries, were observed whereas in the case of the RI only twin boundaries (twin planes (112), well known to be responsible for plasticity in BCC metals) were observed. In the case of the TI, a very distinct $2 \mathrm{NN}$ type BCC divacancy defect was observed and no twin boundaries were seen. It appears that the BCC vacancies arise from dislocation reactions. Clearly, the simplified RI representation of the tool failed to capture the BCC vacancies, whereas the TI failed to capture twin boundaries. These observations of differences in plasticity can help explain the difference in the measured load-displacement $(P-h)$ 
Pre-print (Computational Materials Science) - accepted in April 2018

profile of each indenter type discussed below. These observations on dislocation nucleation in tungsten are a result of the ABOP potential employed in this study. A promising future research direction could be to parametrise carbon with tungsten using more appropriate potential functions, and then revisit these observations on dislocation nucleation behaviour in tungsten during its nanoindentation $[39,50,51]$.

\subsection{Depth variation of nanoindentation hardness and elastic modulus}

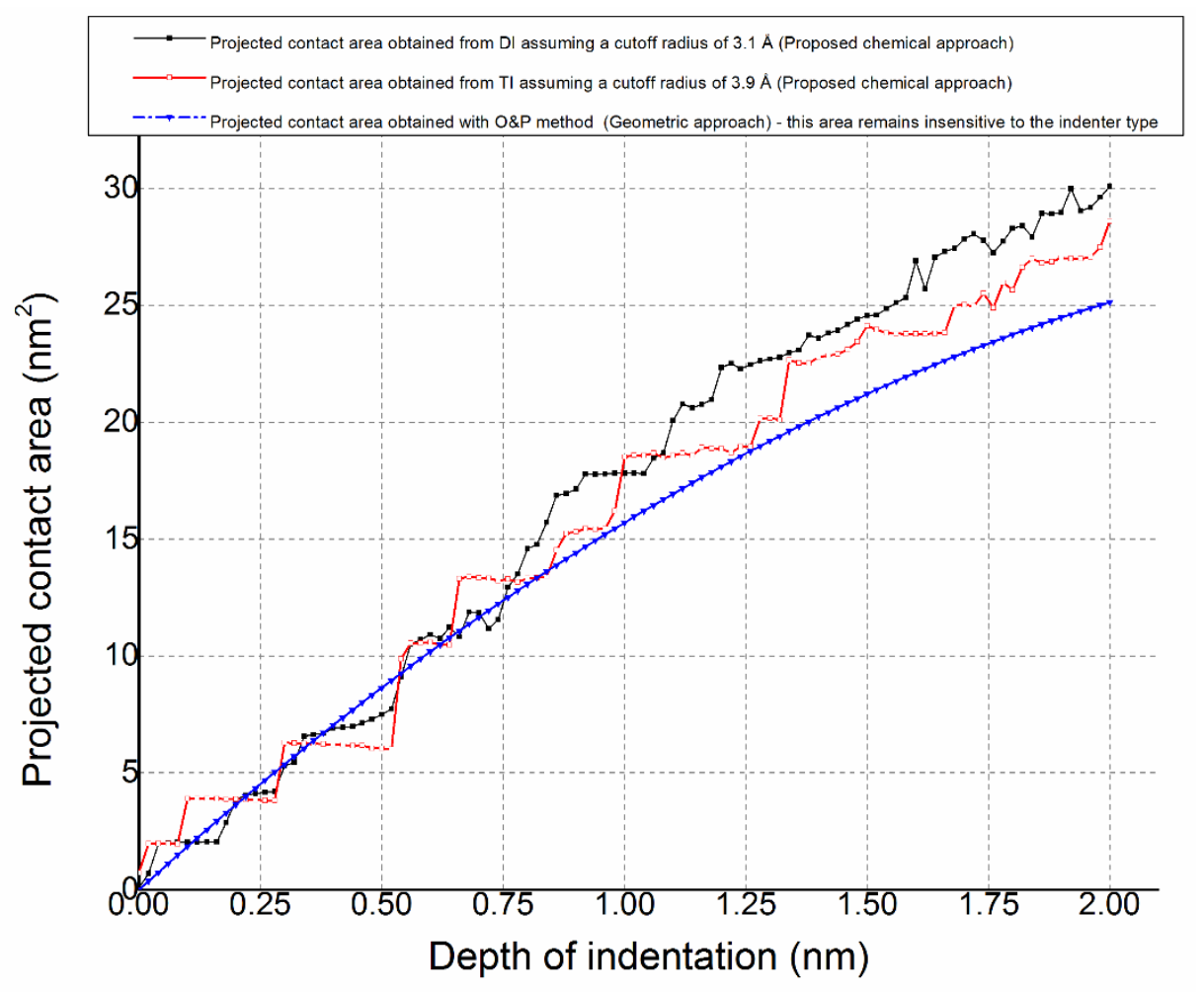




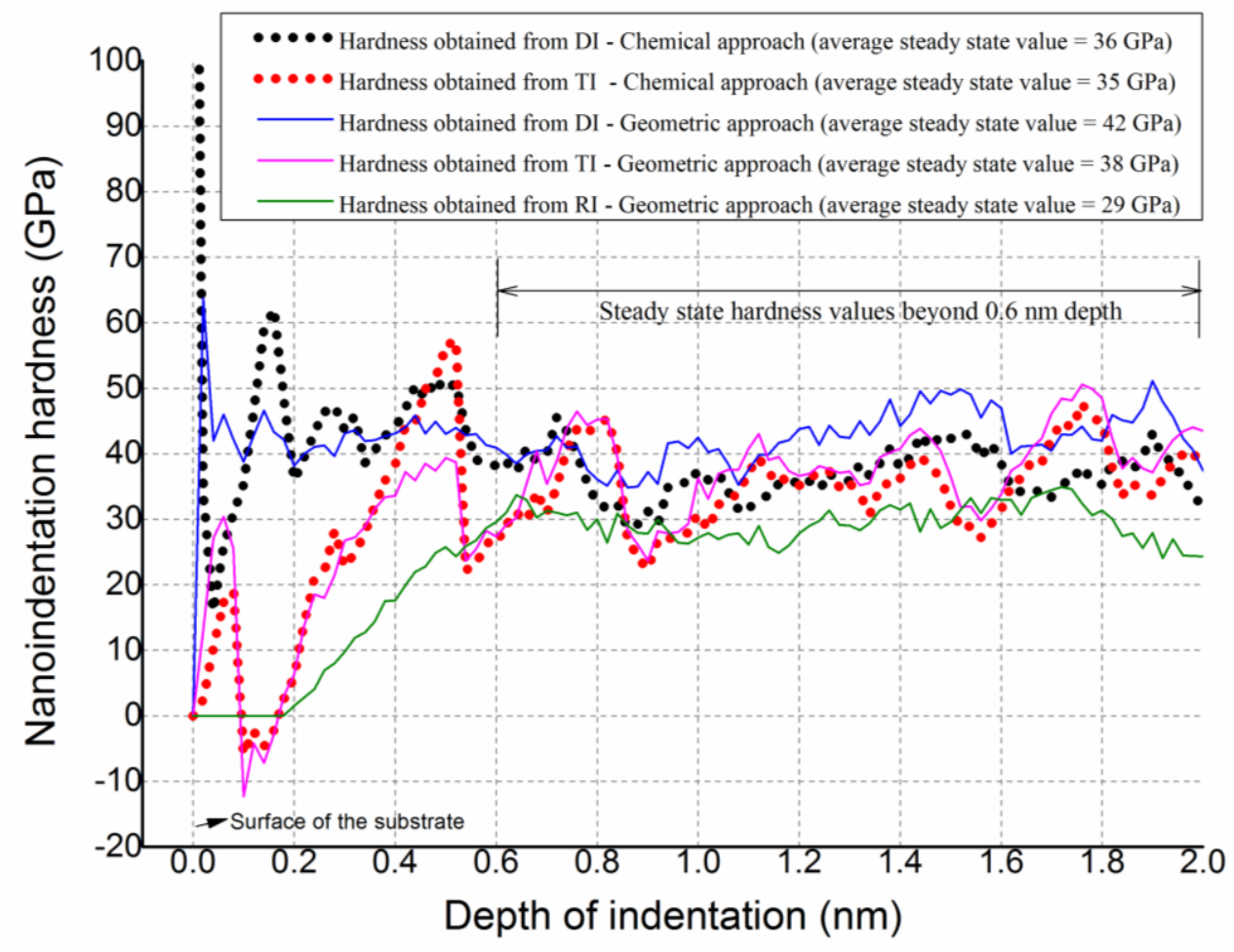

Figure 6: (a) Variation in the projected contact area $\left(\mathrm{nm}^{2}\right)$ with the indentation depth (nm) and (b) variation in the nanoindentation hardness $(\mathrm{GPa})$ obtained from the indentation data of MD simulation for cases where chemical and geometric based approaches are used. The hardness values or the Hertzian stress underneath the indenter achieved steady state in all the three cases only after the indenter has moved into the substrate by about $0.6 \mathrm{~nm}$ [38]

The $P$ - $h$ profile data obtained from nanoindentation was also used to plot the variation in the projected contact area obtained from the proposed chemical based approach as well as the traditional geometric method. The variation in the projected contact areas obtained from the simulation data is shown in figure 6(a). The figure shows that the estimated projected area from the geometric approach is lower than that estimated via the chemical-based approach as it requires an additional correction for the material pile up. Also, interestingly, the projected contact area obtained from TI exhibited a stair-case like pattern which signified its yielding behaviour in certain cases as was highlighted earlier in the literature review. Figure 6(b) shows the variation in the hardness (or contact pressure) with respect to the change in the indentation depth. As for the chemical approach using the DI, figure 6(b) shows that in the beginning, the penetration of the indenter into the substrate leads to a rearrangement of local atomic bonds in the substrate and a smaller contact area, leading to a high jump in the hardness. 
Pre-print (Computational Materials Science) - accepted in April 2018

It is only after $0.6 \mathrm{~nm}$ of indentation depth that some reasonable (albeit very high) values of stresses can be obtained for tungsten. Also, during the approach of the TI to the substrate, the cohesion causes a jump-to-contact leading to a negative compression (hardness) value. It is worth noting that both the geometric and the chemical contact areas revealed very similar values (within an acceptable error margin) signifying the importance of both the methods in the MD simulation framework. In general, the geometric approach provides a higher hardness compared to the chemical approach but despite this, RI provided a lower estimate of the hardness value compared to that provided by the DI and TI. It may also be noted that the MD simulation assumed an atomically flat sample (without any roughness effect contributing to differences in pile-up) so the subtle differences observed are purely material related and would still be relevant when the manufacturing accuracy approaches the atomic length scale.

Also, RI doesn't account for any chemical cohesion activity between the substrate and the indenter, and therefore, RI remains neutral to the substrate exerting pure repulsion. Overall, across all three cases, DI showed the highest (much closer to TI) values of hardness whereas the value obtained by RI was much lower than that in the other two cases.

Next, the O\&P method was applied to obtain the value of elastic modulus $E$ and reduced modulus $E_{r}$ for an indentation depth of $2 \mathrm{~nm}$ to compare different indenters. To compare the elastic modulus computed from the MD simulation against the experiments, we used experimental data readily available at $300 \mathrm{~K}$ with a diamond indenter to extrapolate it at $10 \mathrm{~K}$ for a rigid indenter and compare it with the simulation results shown in figure 7. 
Pre-print (Computational Materials Science) - accepted in April 2018

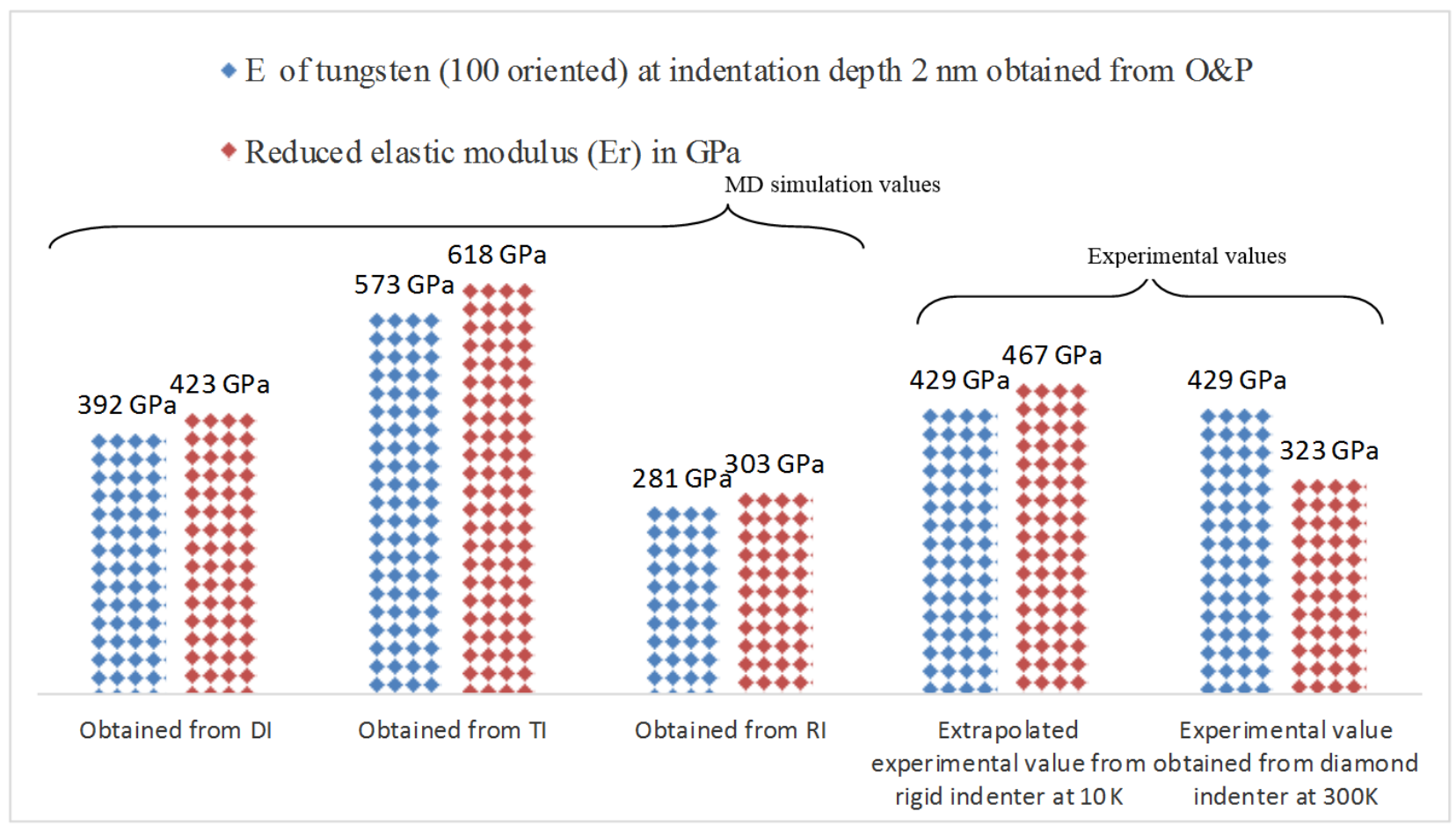

(a)

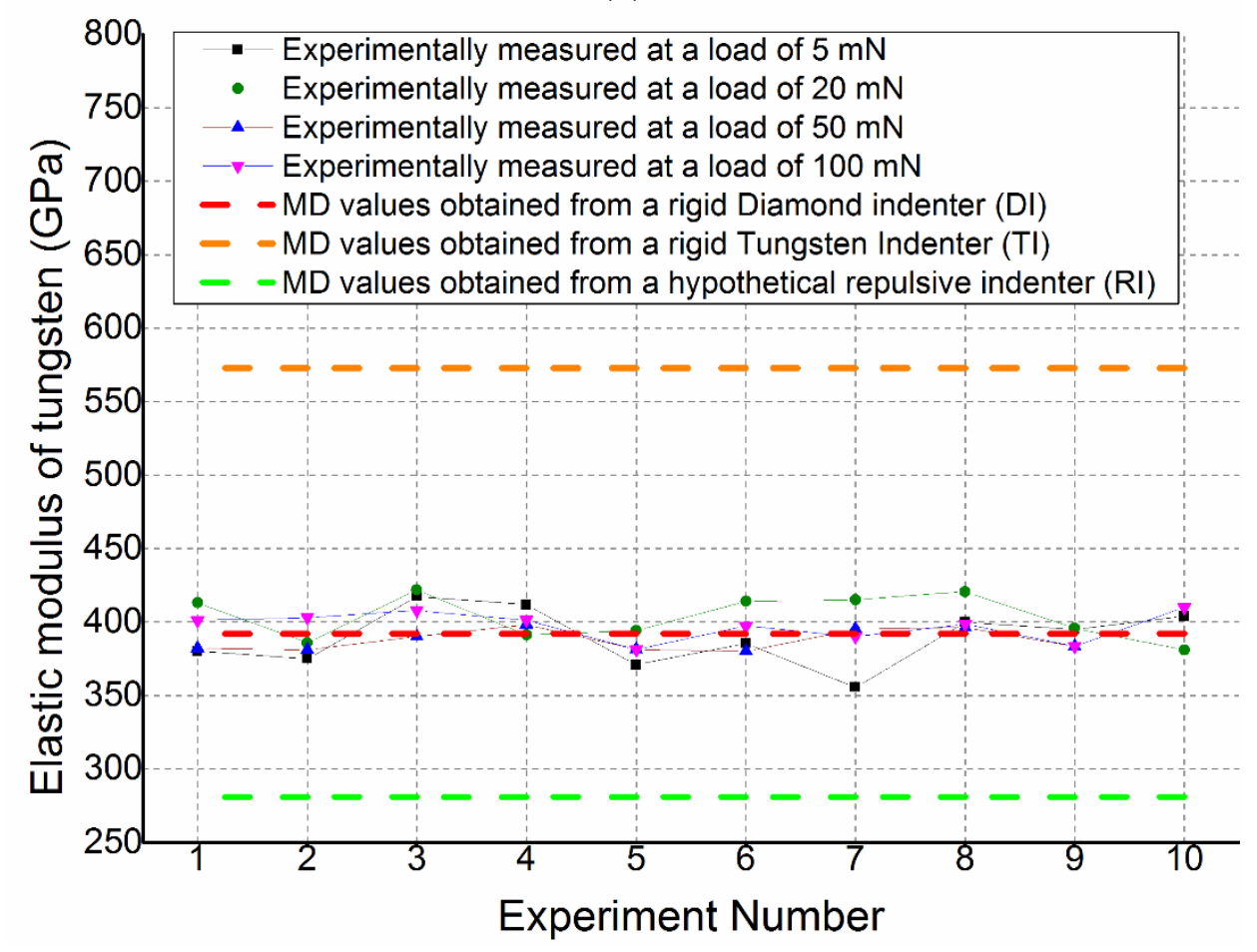

(b)

Figure 7: (a) Value of ' $E$ ' and ' $E_{r}$ ' obtained from MD simulation for three different type of indenters at $10 \mathrm{~K}$ compared with the experimental values (at $300 \mathrm{~K}$ and $10 \mathrm{~K}$ ) obtained from the diamond indenter and a rigid indenter (imaginary) by applying O\&P method at an indentation depth of $2 \mathrm{~nm}$. For MD simulation calculations in all the three cases and for the experimental calculation at $10 \mathrm{~K}$, the value of $E_{\text {indenter }}$ is assumed as infinity due to rigid behavior (b) Statistical comparison of elastic modulus obtained from load-controlled experimental nanoindentation tests against displacementcontrolled MD simulations showing a good correlation between experiments and the MD values obtained from the DI. 
From figure 7(a), it may be observed that the values of $E_{r}$ and ' $E$ ' both vary considerably. For instance, the value of $E_{r}$ with DI (assuming rigid) was obtained as $423 \mathrm{GPa}$ as opposed to an experimental value of $467 \mathrm{GPa}$ (assuming a rigid experimental indenter), while TI and RI revealed this value to be about $618 \mathrm{GPa}$ and $303 \mathrm{GPa}$ respectively. Likewise, the value of ' $E$ ' obtained from the DI was 392 $\mathrm{GPa}$, close enough to the experimental values obtained at different loads (see figure $7 \mathrm{~b}$ ) while the value obtained from TI and RI were $573 \mathrm{GPa}$ (reasonably overestimated) and $281 \mathrm{GPa}$ (heavily underestimated) respectively. It may also be noted here that the value of $E<E_{r}$ in the simulations, while during experiments (with a non-rigid indenter), $E>E_{r}$. This difference appears because the value of $E_{\text {indenter }}$ is assumed to be infinite in the simulations. Since the indenters are considered rigid, a subtractive component of the O\&P equation (which always has a finite magnitude in experiments) becomes zero in case of the rigid indenters, making the value of $E$ obtained from the simulation more relevant than $E_{r}$ for comparing with the experiments.

\subsection{Cyclic nanoindentation at multiple depths}

To explore our results further, we performed cyclic nanoindentation at different depths with the rigid DI (full interactions). A rigid DI was used to perform cyclic nanoindentation trials at finite indentation depths of $0.5 \mathrm{~nm}, 1 \mathrm{~nm}, 1.5 \mathrm{~nm}$ and $2 \mathrm{~nm}$ at a temperature of $10 \mathrm{~K}$. Figure 8 shows the variation in the $P$ - $h$ profiles. The overlapping in the loading profile provides a strong robustness check for the MD simulation results. Even at a small indentation depth of $0.5 \mathrm{~nm}$, the $P-h$ profile shows some residual plasticity. Four indentation depths were chosen to evaluate the unloading slopes at different unloading depths which helped to obtain the nanoindentation hardness and reduced elastic modulus of the tungsten substrate at various indentation depths as shown in figure 9. Figure 8 also shows that the unloading slope (stiffness) obtained for the different $P$ - $h$ plots differs significantly, suggesting that the same value of slope should be used to calculate the elastic modulus at different indentation depths. 


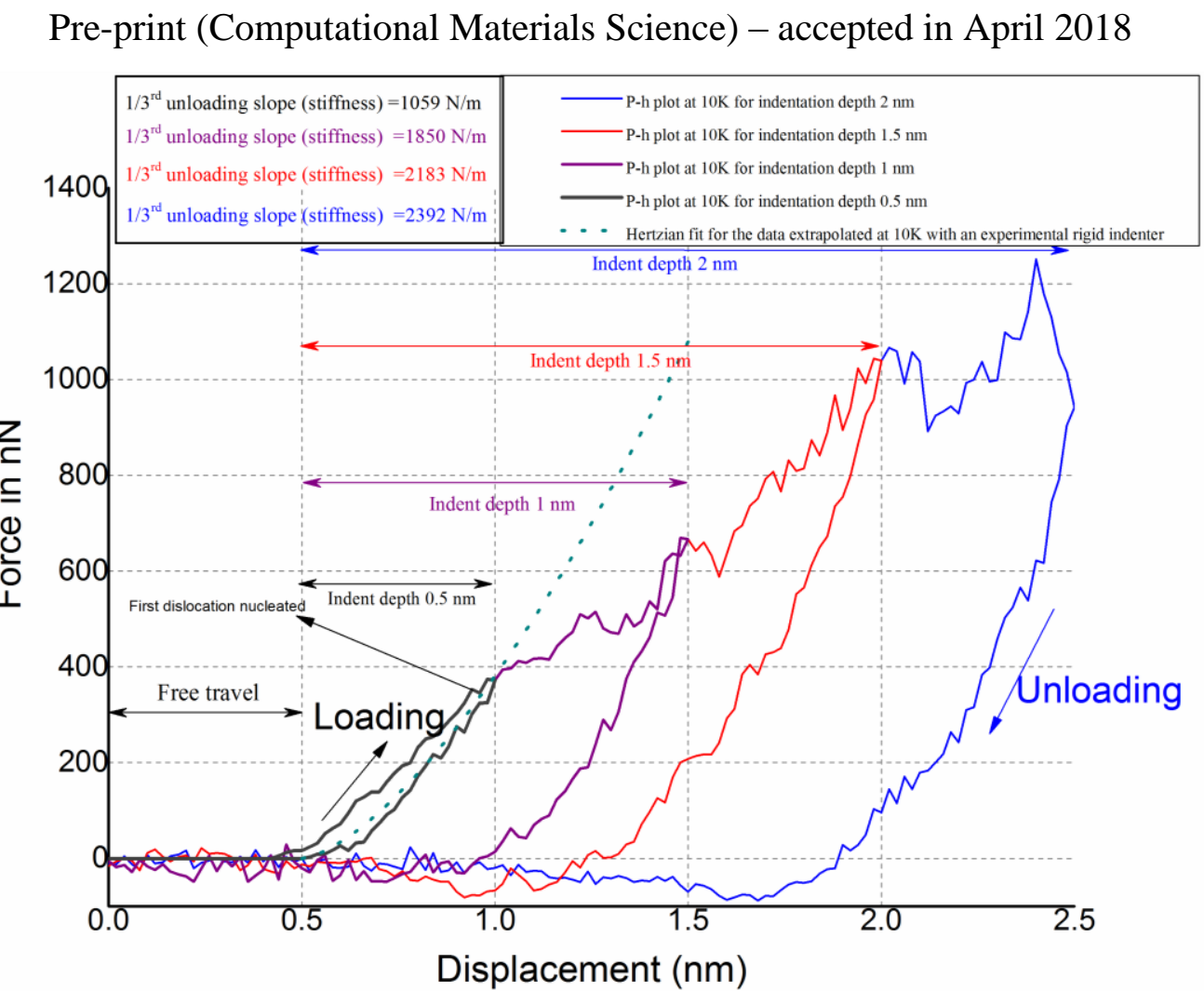

Figure 8: $P$ - $h$ plots obtained at $10 \mathrm{~K}$ using rigid DI for indentation depths of $0.5 \mathrm{~nm}, 1 \mathrm{~nm}, 1.5 \mathrm{~nm}$ and $2 \mathrm{~nm}$ obtained by performing cyclic nanoindentation tests fitted with experimental equivalent Hertz model

For an indentation depth of $2 \mathrm{~nm}$, the unloading slope (stiffness) was obtained as $2392 \mathrm{~N} / \mathrm{m}$ as opposed to $1059 \mathrm{~N} / \mathrm{m}$ for an indentation depth of $0.5 \mathrm{~nm}$. Also, the peak load and the projected contact area differed for different indentation depths. These data were used as an input to obtain the reduced modulus and hardness shown in figure 9. Figure 9 shows that a strong extrinsic size effect is present. For instance, at a shallow indentation depth of $0.5 \mathrm{~nm}$, the reduced modulus was very low $(\sim 320$ $\mathrm{GPa}$ ), but as the indentation depth increased, the value saturated to about $420 \mathrm{GPa}$. Similarly, the value of nanoindentation hardness fluctuated between 40 and $44 \mathrm{GPa}$ except for the indentation depth of $1.5 \mathrm{~nm}$ where the nanoindentation hardness was of the order of $49 \mathrm{GPa}$. 


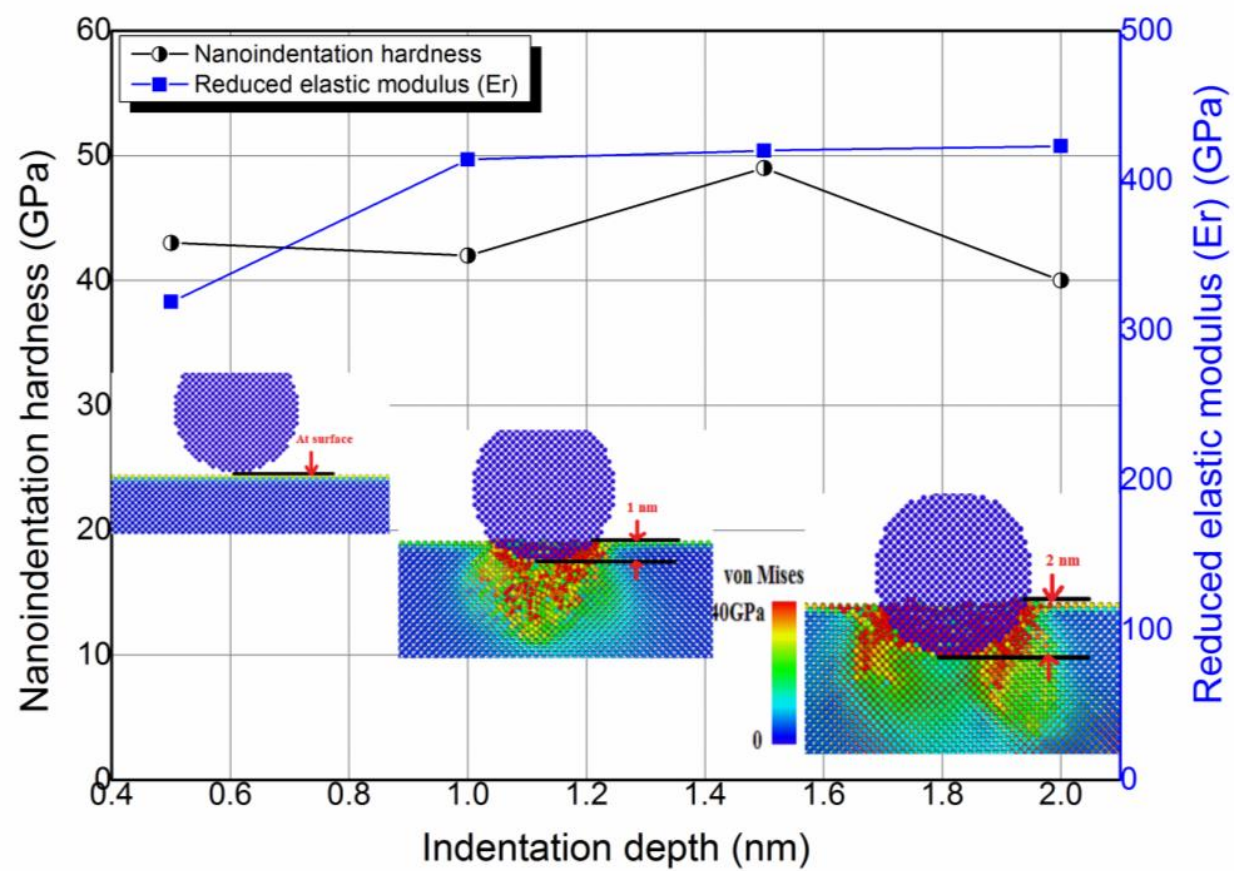

Figure 9: Variation in the instantaneous nanoindentation hardness (GPa) and reduced elastic modulus $\left(E_{r}\right)$ of the tungsten substrate obtained by applying O\&P method in conjunction with the $P$ - $h$ plots obtained at depths $0.5 \mathrm{~nm}, 1 \mathrm{~nm}, 1.5 \mathrm{~nm}$ and $2 \mathrm{~nm}$ respectively

Figure 9 also shows the variation in the von Mises stress with the indentation depth in the atomic framework. At a small indentation depth of $1 \mathrm{~nm}$, the von Mises stress remained focused underneath the indenter (signifying compression) and then moves on to become tensile at the periphery of the indenter leading to pile-up of the material.

Finally, stress tensor information was also used to obtain information on the nanoscale yielding in tungsten. This was done by converting the atomic stress tensor to a physical stress tensor which was then used to calculate Major Principal stress, Minor Principal stress, Tresca stress, von Mises stress and Octahedral shear stress [52]. Table VI shows the yielding stress indicators of tungsten in the deformation zone (in conjunction with figure $1 \mathrm{~b}$ ) in all three cases. It can be seen that the Tresca stress and von-Mises stress (more appropriate for predicting the yielding of ductile materials) were similar for the DI and TI but differed for the RI, suggesting that the nanoindentation performed by an RI provides an overestimated value of the nanoscale yielding stress for tungsten. Experimentally, the mean contact pressure $\left(p_{m}\right)$ is obtained as 0.667 times the maximum contact pressure in Hertzian spherical contact and for the smallest $R$ values of the indenter, a value of $(33+/-5)$ GPa for the (100) 
Pre-print (Computational Materials Science) - accepted in April 2018

oriented $W$ was obtained, close to what is obtained from the MD for DI and TI and to an extent with RI too.

Table VI: Nanoscale yielding stresses of tungsten obtained from the MD simulation

\begin{tabular}{|l|c|c|c|}
\hline Stress peak (GPa) causing nanoscale yielding of tungsten & Rigid DI & Rigid TI & Rigid RI \\
\hline Von-Mises stress & 30 & 30 & 42 \\
\hline Octahedral shear stress & 14 & 14 & 20 \\
\hline Tresca stress & 17 & 17 & 21 \\
\hline Major principal stress & -81 & -53 & -77 \\
\hline Minor principal stress & -47 & -19 & -34 \\
\hline Hydrostatic stress & -62 & -35 & -49 \\
\hline
\end{tabular}

A substantive variation was observed in the Major, Minor and hydrostatic stress between the three cases, and the immediate reason for these differences was not clear. The large variations in the principal stresses could possibly be attributed to the cohesive dynamics between the two kinds of atoms. It may be recalled that a purely repulsive indenter repels all the atoms away so there is no cohesive force between the tungsten atoms. In the TI case, this anomaly arises from Van der Waals forces between the two kinds of atoms. It is already known that the surface forces are dominated by Van der Waals forces [53]. At interfaces (during approach), the adhesion between the diamond and the tungsten atoms is mainly due to Van der Waals forces whereas in the case of TI, this adhesion is replaced by cohesion which might be responsible for the observed difference. It may also be noted that while the difference in the critical stresses for DI and TI may look quite large at the first glance, RI being a repulsive indenter exerts a purely repulsive force (along the radial direction) and hence the friction between the indenter and the substrate surface is disregarded. In our view, these differences indicate that the von-Mises and Tresca criterion are more suitable for prediction of nanoscale yielding of metals than the principal stress criterion which was observed to be more appropriate for brittle semi conductive material such as silicon [52]. 
Pre-print (Computational Materials Science) - accepted in April 2018

\section{Scale differences between MD simulation and experiments}

MD scales differ in the timescale probed in nanoindentation experiments and, with some exceptions, the length scale. As such, MD simulation results cannot be compared directly with experimental nanoindentation results. Beside differing in the time scale (MD observations are in pico-nano seconds as opposed to the experimental observations seen at the microsecond scale), the presence of crystal defects in the real-world materials is always inherent whereas a perfect crystal model as in an MD simulation always assumes an activation energy barrier. Also, at an experimental scale, the size of the indenter is substantially larger, leading to activation of several dislocations to create a dislocation burst at yield. In practice, the stress at which this happens is dependent on the pre-existing dislocation density, nanoscale surface roughness, residual stress, the testing environment, and indenter size, all of which can affect the probability of encountering dislocations in a given volume. At the MD scale, a highly-localised yield is affected rather than a burst and a dislocation free ideal crystal is the starting configuration in the presence of vacuum. Overall, MD simulations are based on materials having a specific theoretical strength, whereas in experiments, this can vary stochastically.

Furthermore, two key assumptions of the O\&P method are: 1) Unloading is purely elastic, and 2) The elastic unloading relaxation involves only vertical displacements (Mott assumption). Our simulation involves an indentation tip consisting of an atomic structure that is chemically active in terms of interaction with the substrate, but the positions of the atoms are completely fixed and the elastic or plastic relaxation of the tip is not allowed. However, the substrate (tungsten) atoms are both chemically active and mobile under all interaction forces and can exhibit elastic and plastic relaxation. In principle they can also diffuse, but this is unlikely in tungsten at $10 \mathrm{~K}$ and over short simulation timescales. All these aspects count towards making the MD simulation findings not directly comparable to the experiments, but complimentary.

Interestingly, it was suggested earlier that if $h_{f} / h_{\max }<0.7$, then the material should show sink-in behaviour or for a value higher than 0.7 pile-up dominates $[37,54]$. The MD results in this work based on the $P-h$ plots revealed the $h_{f} / h_{\max }$ ratio to be 0.69 for DI and RI and 0.78 for the TI. The 
Pre-print (Computational Materials Science) - accepted in April 2018

simulation video shows a boundary marking the transition to pile-up. This is another interesting area worth pursuing further: Extrinsic size effects at the nanoscale are not very well understood currently, although, experimentally, pure tungsten is reported to show strong pile-up behaviour at experimental scales similar to other BCC metals [55].

\section{Conclusion}

Driven by the notion that diamond is an ultra-hard material and will undergo negligible elastic or plastic transition during the nanoindentation process, many of the previously published MD simulation studies assume the indenter is an ideally rigid material. This assumption has been implemented by following either of the three cases: (a) by assuming a rigid diamond indenter (DI), (b) by assuming a rigid indenter comprising of the atoms of the same material of the substrate and (c) by assuming an imaginary spherical rigid repulsive indenter described by a force of magnitude $F(r)$ $=-\mathrm{K}(\mathrm{r}-\mathrm{R})^{2}$ where $K$ is the force constant and $R$ is the radius of the indenter (RI). However, previous studies have not revealed how these assumptions compare with each other and what would be the implications of such assumptions. This work attempts to address this scientific problem by comparing the material response of single crystal tungsten during nanoindentation by using the aforementioned indenter choices. Indentation hardness and elastic modulus obtained from the simulation revealed different values with individual choices which were mapped against the experiments. Based on these observations, the following can be concluded:

(i) Even when an indenter is considered rigid, the near-surface phenomena such as cohesion or adhesion are always active, and this can trigger changes in the way plasticity is induced in the substrate. This testbed study on tungsten showed that a purely repulsive indenter (RI) implemented by a hypothetical force potential results in an underestimated value of hardness and elastic modulus compared to that using a diamond indenter. On the other hand, an assumption of the rigid indenter material consisting of the same atoms (tungsten in this work referred to as TI) to that of the 
Pre-print (Computational Materials Science) - accepted in April 2018

substrate atoms resulted in an overestimated value of hardness and modulus measurements. While the estimates of the modulus and hardness from the hard indenter choices (either pure repulsive or consisting of the same substrate atoms) can take any order depending on the material being indented, it is advisable in general for future nanoindentation studies to use potentials which are fully parameterised for carbon (to model a diamond indenter) and for the respective substrate materials.

(ii) An automated dislocation extraction algorithm showed $\mathbf{b}=1 / 2\langle 111\rangle$ and $\mathbf{b}=\langle 100\rangle$ type prismatic dislocation loops to be responsible for the nanoscale plasticity in tungsten. The volumetric dislocation density defined as total length of dislocations per unit plastic volume were found to be in the range of $2 \times 10^{16} \mathbf{~ m}^{-2}$ to $3 \times 10^{16} \mathbf{m}^{-2}$.

(iii) The microstructure underneath the deformation zone of the substrate showed BCC vacancy while indenting with a rigid diamond indenter, BCC divacancy (2nd NN type) while using a rigid tungsten indenter and twin boundaries while using a purely repulsive indenter. The formation of prismatic dislocation loops underneath the indenter in conjunction with the twin planes in $\mathrm{W}$ is directly analogous to similar previous findings for BCC iron[56].

(iv) In accordance with the macroscopic theory on yielding in materials, this work revealed that the von Mises and Tresca stress criterion were found to be more appropriate for predicting the yielding in tungsten even at the nanoscale as opposed to brittle silicon where the Minor Principal stress criterion was observed to be a more robust indicator of yielding [52].

(v) This research work compared the projected contact area using geometric (Oliver and Pharr method) and chemical criteria. Both the geometric and chemical contact areas were similar (within an acceptable error margin) signifying the importance of both methods in the MD simulation framework. In general, the estimated projected area from the geometric approach is lower than that estimated via the chemical-based 
Pre-print (Computational Materials Science) - accepted in April 2018

approach as it requires an additional correction for the material pile up. Consequently, the geometric approach indicates a higher hardness compared to the chemical approach. The difference however originates purely from the material properties because the MD simulation assumed an atomically flat sample (without any roughness effect contributing to differences in pile-up) so the subtle differences would still be very relevant when the manufacturing accuracy approaches atomic length scale.

Based on these results, it can be concluded that the choice of indenter in the MD simulation (even if the indenter is considered perfectly rigid) should not be made on an arbitrary basis but after a careful assessment.

\section{Acknowledgments:}

This work was done in the Centre for Doctoral Training in Ultra-Precision at Cranfield University which is supported by the RCUK via Grant No.: EP/K503241/1. Authors acknowledge help of Dr Michael Knaggs for accessing Cranfield's Delta HPC service as well as PRACE for granting access to HLRS resource based in Germany at Stuttgart. SG and EG acknowledge STSM support from the European COST Action CA15102 and COST Action MP1303of the Horizon 2020.

\section{References}

1. Mo, Y., Turner, K.T., and Szlufarska, I., Friction laws at the nanoscale. Nature, 2009. 457(7233): p. 1116-1119.

2. Belak, J., Nanotribology: Modelling Atoms When Surfaces Collide, in Energy and Technology Review. 1994, Lawrence Livermore Laboratory.

3. Zykova-Timan, T., Ceresoli, D., and Tosatti, E., Peak effect versus skating in hightemperature nanofriction. Nature Materials, 2007. 6(3): p. 230-234.

4. Goel, S., A topical review on "The current understanding on the diamond machining of silicon carbide". Journal of Physics D: Applied Physics, 2014. 47(24): p. 243001.

5. Landman, U., Luedtke, W.D., Burnham, N.A., and Colton, R.J., Atomistic Mechanisms and Dynamics of Adhesion, Nanoindentation, and Fracture. Science, 1990. 248(4954): p. 454- 
461.

6. Bhushan, B., Israelachvili, J.N., and Landman, U., Nanotribology: friction, wear and lubrication at the atomic scale. Nature, 1995. 374(6523): p. 607-616.

7. Hagelaar, J., Bitzek, E., Flipse, C., and Gumbsch, P., Atomistic simulations of the formation and destruction of nanoindentation contacts in tungsten. Physical Review B, 2006. 73(4): p. 045425.

8. Komanduri, R., Chandrasekaran, N., and Raff, L.M., Molecular dynamics simulation of atomic-scale friction. Physical Review B, 2000. 61(20): p. 14007-14019.

9. Lodes, M.A., Hartmaier, A., Göken, M., and Durst, K., Influence of dislocation density on the pop-in behavior and indentation size effect in CaF2 single crystals: Experiments and molecular dynamics simulations. Acta Materialia, 2011. 59(11): p. 4264-4273.

10. Pfetzing-Micklich, J., Somsen, C., Dlouhy, A., Begau, C., Hartmaier, A., Wagner, M.F.X., and Eggeler, G., On the crystallographic anisotropy of nanoindentation in pseudoelastic NiTi. Acta Materialia, 2013. 61(2): p. 602-616.

11. Kelchner, C.L., Plimpton, S.J., and Hamilton, J.C., Dislocation nucleation and defect structure during surface indentation. Physical Review B, 1998. 58(17): p. 11085.

12. Wang, B., Zhang, Z., Cui, J., Jiang, N., Lyu, J., Chen, G., Wang, J., Liu, Z., Yu, J., Lin, C., Ye, F., and Guo, D., In Situ TEM Study of Interaction between Dislocations and a Single Nanotwin under Nanoindentation. ACS Applied Materials \& Interfaces, 2017. 9(35): p. 29451-29456.

13. Zhang, Z., Wang, B., Kang, R., Zhang, B., and Guo, D., Changes in surface layer of silicon wafers from diamond scratching. CIRP Annals-Manufacturing Technology, 2015. 64(1): p. 349-352.

14. Smithells, C.J., Metals reference book. Vol. 3. 1967: Butterworth.

15. Zhang, J.-M., Zhang, Y., Xu, K.-W., and Ji, V., Representation surfaces of Young's modulus and Poisson's ratio for BCC transition metals. Physica B: Condensed Matter, 2007. 390(1): p. 106-111.

16. Rieth, M., Dudarev, S., Gonzalez de Vicente, S., Aktaa, J., Ahlgren, T., Antusch, S., Armstrong, D., Balden, M., Baluc, N., and Barthe, M.-F., A brief summary of the progress on the EFDA tungsten materials program. Journal of Nuclear Materials, 2013.

17. Huang, H., Wu, Y., Wang, S., He, Y., Zou, J., Huang, B., and Liu, C., Mechanical properties of single crystal tungsten microwhiskers characterized by nanoindentation. Materials Science and Engineering: A, 2009. 523(1): p. 193-198.

18. Roundy, D., Krenn, C., Cohen, M.L., and Morris Jr, J., The ideal strength of tungsten. Philosophical Magazine A, 2001. 81(7): p. 1725-1747.

19. Bahr, D.F., Kramer, D.E., and Gerberich, W.W., Non-linear deformation mechanisms during nanoindentation. Acta Materialia, 1998. 46(10): p. 3605-3617.

20. Lassner, E. and Schubert, W.-D., Tungsten: properties, chemistry, technology of the elements, alloys, and chemical compounds. 1999: Springer.

21. Argon, A.S. and Maloof, S.R., Plastic deformation of tungsten single crystals at low temperatures. Acta Metallurgica, 1966. 14(11): p. 1449-1462.

22. Yao, W.Z., Krill, C.E., III, Albinski, B., Schneider, H.C., and You, J.H., Plastic material parameters and plastic anisotropy of tungsten single crystal: a spherical micro-indentation study. Journal of Materials Science, 2014. 49(10): p. 3705-3715.

23. Beake, B.D. and Goel, S., Incipient plasticity in tungsten during nanoindentation: Dependence on surface roughness, probe radius and crystal orientation. International Journal of Refractory Metals and Hard Materials, 2018. 75: p. 63-69.

24. Plimpton, S., Fast Parallel Algorithms for Short-Range Molecular Dynamics. Journal of Computational Physics, 1995. 117: p. 1-19.

25. Stukowski, A., Visualization and analysis of atomistic simulation data with OVITO-the Open Visualization Tool. Modelling and Simulation in Materials Science and Engineering, 2010. 18(1).

26. Stukowski, A., Bulatov, V.V., and Arsenlis, A., Automated identification and indexing of 
Pre-print (Computational Materials Science) - accepted in April 2018

dislocations in crystal interfaces. Modelling and Simulation in Materials Science and Engineering, 2012. 20(8): p. 085007.

27. Stukowski, A. and Albe, K., Extracting dislocations and non-dislocation crystal defects from atomistic simulation data. Modelling and Simulation in Materials Science and Engineering, 2010. 18(8): p. 085001.

28. Goel, S., Faisal, N.H., Luo, X., Yan, J., and Agrawal, A., Nanoindentation of polysilicon and single crystal silicon: Molecular dynamics simulation and experimental validation. Journal of Physics D: Applied Physics, 2014. 47(27): p. 275304.

29. Goel, S., Luo, X., Agrawal, A., and Reuben, R.L., Diamond machining of silicon: A review of advances in molecular dynamics simulation. International Journal of Machine Tools and Manufacture, 2015. 88(0): p. 131-164.

30. Gröger, R., Bailey, A., and Vitek, V., Multiscale modeling of plastic deformation of molybdenum and tungsten: I. Atomistic studies of the core structure and glide of $1 / 2\langle 111\rangle$ screw dislocations at OK. Acta Materialia, 2008. 56(19): p. 5401-5411.

31. Tersoff, J., New empirical approach for the structure and energy of covalent systems. Physical Review B, 1988. 37(12): p. 6991.

32. Tersoff, J., Empirical interatomic potential for silicon with improved elastic properties. Physical Review B, 1988. 38(14): p. 9902.

33. Juslin, N., Erhart, P., Träskelin, P., Nord, J., Henriksson, K.O., Nordlund, K., Salonen, E., and Albe, K., Analytical interatomic potential for modeling nonequilibrium processes in the $W-$ $C-H$ system. Journal of Applied Physics, 2005. 98(12): p. 123520.

34. Oliver, W.C. and Pharr, G.M., Improved technique for determining hardness and elastic modulus using load and displacement sensing indentation experiments. Journal of Materials Research, 1992. 7(6): p. 1564-1583.

35. Ziegenhain, G., Urbassek, H.M., and Hartmaier, A., Influence of crystal anisotropy on elastic deformation and onset of plasticity in nanoindentation: a simulational study. Journal of Applied Physics, 2010. 107(6): p. 061807.

36. Fischer-Cripps, A.C., Nanoindentation Testing, in Nanoindentation. 2011, Springer New York: New York, NY. p. 21-37.

37. Herbert, E., Pharr, G., Oliver, W., Lucas, B., and Hay, J., On the measurement of stress-strain curves by spherical indentation. Thin Solid Films, 2001. 398: p. 331-335.

38. Chen, S. and Ke, F., MD simulation of the effect of contact area and tip radius on nanoindentation. Science in China Series G: Physics, Mechanics and Astronomy, 2004. 47(1): p. 101-112.

39. Cereceda, D., Stukowski, A., Gilbert, M., Queyreau, S., Ventelon, L., Marinica, M., Perlado, J., and Marian, J., Assessment of interatomic potentials for atomistic analysis of static and dynamic properties of screw dislocations in W. Journal of Physics: Condensed Matter, 2013. 25(8): p. 085702.

40. Finnis, M.W. and Sinclair, J.E., A simple empirical N-body potential for transition metals. Philosophical Magazine A, 1984. 50(1): p. 45-55.

41. Pastewka, L., Klemenz, A., Gumbsch, P., and Moseler, M., Screened empirical bond-order potentials for Si-C. Physical Review B, 2013. 87(20): p. 205410.

42. Hertzberg, R.W., Deformation and fracture mechanics of engineering materials. Vol. 89. 1996: Wiley New York.

43. Goel, S., Stukowski, A., Luo, X., Agrawal, A., and Reuben, R.L., Anisotropy of single-crystal $3 C-S i C$ during nanometric cutting. Modelling and Simulation in Materials Science and Engineering, 2013. 21(6): p. 065004.

44. Yamakov, V.I., Warner, D.H., Zamora, R.J., Saether, E., Curtin, W.A., and Glaessgen, E.H., Investigation of crack tip dislocation emission in aluminum using multiscale molecular dynamics simulation and continuum modeling. Journal of the Mechanics and Physics of Solids, 2014. 65(0): p. 35-53.

45. Hill, R., The Elastic Behaviour of a Crystalline Aggregate. Proceedings of the Physical 
Society. Section A, 1952. 65(5): p. 349.

46. Ni, W., Cheng, Y.-T., Cheng, C.-M., and Grummon, D.S., An energy-based method for analyzing instrumented spherical indentation experiments. Journal of materials research, 2004. 19(01): p. 149-157.

47. Klemenz, A., Gola, A., Moseler, M., and Pastewka, L., Contact mechanics of graphenecovered metal surfaces. Applied Physics Letters, 2018. 112(6): p. 061601.

48. Cross, G.L., Schirmeisen, A., Grütter, P., and Dürig, U.T., Plasticity, healing and shakedown in sharp-asperity nanoindentation. Nature materials, 2006. 5(5): p. 370-376.

49. Wang, B., Gao, Y., and Urbassek, H.M., Microstructure and magnetic disorder induced by nanoindentation in single-crystalline Fe. Physical Review B, 2014. 89(10): p. 104105.

50. Goel, S. and Stukowski, A., Comment on "Incipient plasticity of diamond during nanoindentation" by C. Xu, C. Liu and H. Wang, RSC Advances, 2017, 7, 36093. RSC Advances, 2018. 8(10): p. 5136-5137.

51. Chavoshi, S.Z., Xu, S., and Goel, S., Addressing the discrepancy of finding the equilibrium melting point of silicon using molecular dynamics simulations. Proceedings of the Royal Society A: Mathematical, Physical and Engineering Science, 2017. 473(2202).

52. Goel, S., Kovalchenko, A., Stukowski, A., and Cross, G., Influence of microstructure on the cutting behaviour of silicon. Acta Materialia, 2016. 105: p. 464-478.

53. DelRio, F.W., de Boer, M.P., Knapp, J.A., Reedy, E.D., Clews, P.J., and Dunn, M.L., The role of van der Waals forces in adhesion of micromachined surfaces. Nature Materials, 2005. 4(8): p. 629-634.

54. Bolshakov, A. and Pharr, G., Influences of pileup on the measurement of mechanical properties by load and depth sensing indentation techniques. Journal of Materials Research, 1998. 13(4): p. 1049-1058.

55. Remington, T., Ruestes, C., Bringa, E., Remington, B., Lu, C., Kad, B., and Meyers, M., Plastic deformation in nanoindentation of tantalum: A new mechanism for prismatic loop formation. Acta Materialia, 2014. 78: p. 378-393.

56. Goel, S., Martinez, F.D., Chavoshi, S.Z., Khatri, N., and Giusca, C., Molecular dynamics simulation of the elliptical vibration assisted machining (EVAM) of pure iron (in press). Journal of Micromanufacturing, Sage publisher, 2018. 